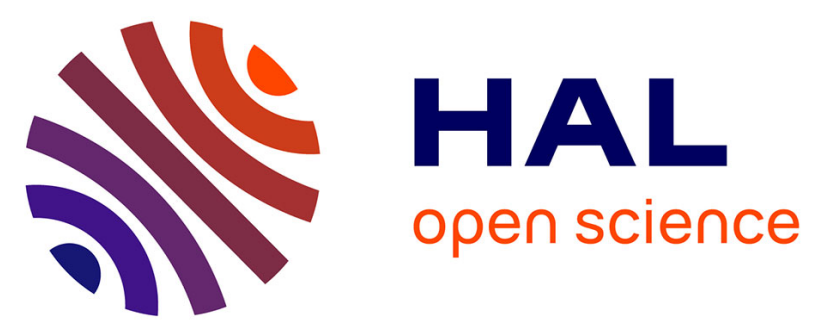

\title{
Multiscale prediction of acoustic properties for glass wools: Computational study and experimental validation
}

\author{
M. He, C. Perrot, J. Guilleminot, P. Leroy, G. Jacqus
}

\section{To cite this version:}

M. He, C. Perrot, J. Guilleminot, P. Leroy, G. Jacqus. Multiscale prediction of acoustic properties for glass wools: Computational study and experimental validation. Journal of the Acoustical Society of America, 2018, 143 (6), pp.3283 - 3299. 10.1121/1.5040479 . hal-01818174

\section{HAL Id: hal-01818174 \\ https://hal.science/hal-01818174}

Submitted on 18 Jun 2018

HAL is a multi-disciplinary open access archive for the deposit and dissemination of scientific research documents, whether they are published or not. The documents may come from teaching and research institutions in France or abroad, or from public or private research centers.
L'archive ouverte pluridisciplinaire HAL, est destinée au dépôt et à la diffusion de documents scientifiques de niveau recherche, publiés ou non, émanant des établissements d'enseignement et de recherche français ou étrangers, des laboratoires publics ou privés. 


\title{
Multiscale prediction of acoustic properties for glass wools: Computational study and experimental validation
}

\author{
M. He and C. Perrot ${ }^{\mathrm{a}}$ \\ Laboratoire Modélisation et Simulation Multi Echelle, \\ MSME UMR 8208 CNRS, Université Paris-Est, \\ 5 bd Descartes, 77454 Marne-la-Vallée, France \\ J. Guilleminot \\ Department of Civil and Environmental Engineering, \\ Pratt School of Engineering, Duke University, Box 9028\%, \\ 121 Hudson Hall, Durham, NC 27708-0287, USA \\ P. Leroy \\ Isover Saint-Gobain CRIR, B.P. 10019, 60291 Rantigny Cedex, France \\ G. Jacqus ${ }^{b}$ \\ CSTB, 84 Avenue Jean Jaures, Champs-sur-Marne, \\ 77447 Marne-la-Vallée Cedex 2, France
}

(Dated: May 16, 2018)

\footnotetext{
acamille.perrot@u-pem.fr; Corresponding author.

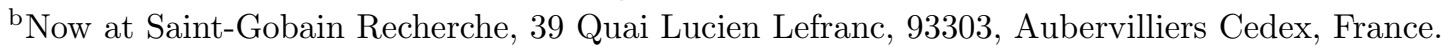




\begin{abstract}
This work is concerned with the multiscale prediction of the transport and sound absorption properties associated with industrial glass wool samples. In the first step, an experimental characterization is performed on various products using optical granulometry and porosity measurements. A morphological analysis, based on scanning electron imaging, is further conducted to identify the probability density functions associated with the fiber angular orientation. The key morphological characterization parameters of the microstructure, which serve as input parameters of the model, include the porosity, the weighted volume diameter accounting for both lengths and diameters of the analyzed fibers (and therefore the specific surface area of the random fibrous material), and the preferred out-of-plane fiber orientation generated by the manufacturing process. A computational framework is subsequently proposed and allows for the reconstruction of an equivalent fibrous network. A fully stochastic microstructural model, parameterized by the probability laws inferred from the database, is also proposed herein. Multiscale simulations are carried out to estimate transport properties and sound absorption. With no adjustable parameter, the results accounting for ten different samples obtained with various processing parameters, are finally compared with the experimental data and used to assess the relevance of the reconstruction procedures and the multiscale computations.
\end{abstract}

PACS numbers: 43.55.Ev, 43.50.Gf 


\section{INTRODUCTION}

What are the microscopic bases of macroscopic properties in acoustic fibrous media? How do macroscopic transport properties (e.g., static viscous and thermal permeabilities, viscous characteristic length and high frequency tortuosity) depend on the microstructural parameters of a fibrous material? These are two of the many questions that have dominated studies of fluid flow and thermal diffusion through microscopically-disordered fibrous materials such as glass wools. Such questions may be addressed in a variety of ways $[1,2]$. Perhaps, the most direct method is to conduct a series of laboratory measurements on samples of varying sizes and types [3-8]. Alternatively, in the quest for theoretical understanding, one may seek to better understand the mathematical or physical basis of the generalized Darcy-scale equations for macroscopic transports [9-14]. Lastly, one can consider studies based on numerical simulations [15-17].

Each of these approaches has strengths and weaknesses. Laboratory measurements are of undisputed value; however, their usefulness may be limited to a specific range of already available manufactured materials. Moreover, laboratory measurements are subjected to measurement noise and potential methodological errors. It is therefore not an absolute reference either. Importantly, the attempts in the literature of fibrous materials where transport properties have been related empirically to microstructural parameters - usually through some kind of power law with fitted exponents - cannot reflect what the microphysical origins are behind exponents modifications; suggesting that this approach is not appropriate to understand transport processes in fibrous materials. Analytical studies, on the other hand, are not necessarily limited to a specific kind of fiber materials, but they usually require simplifying assumptions (e.g., periodic array of cylinders [18], specific fiber orientations $[19,20]$, or negligible interaction between the shear stress fields of neighboring fibers [21]) that have only a partial relevance to reality. Numerical simulation usually attempts to bridge the gap between theory and experiments. It is typically hampered, however, by either the need to simplify geometry $[16,18]$ or physics $[15,22]$. In recent years, however, another approach to the numerical study of diffusion and fluid flow through fibrous media has gained some popularity $[17,23,24]$. The idea is to numerically solve the asymptotic behaviors of the linearized Navier-Stokes and heat equations in a realistic microscopically disordered geometry, and then study how volume-averaged properties of the diffusion process 
and the fluid flow depend on the details of the microstructures. Such studies offer the ability to study the micro-physical basis of macroscopic transport without the need for simplified geometries or physics; they are however limited to samples of small size.

Considering the difficulties that have been stated about empirical and analytical approaches, an attempt to relate fibrous structure to transport parameters should be based on direct measurements of the microstructural parameters of a single fibrous sample. Examples of such microstructural parameters include porosity, specific surface area, and some kind of average or typical fiber diameter and fiber orientation. We could then proceed to numerically solve the appropriate transport equations in a reconstructed fibrous microstructure. A challenge is in choosing the appropriate microstructural parameters and in proposing a reconstruction procedure as a way of predicting transport properties of the sample.

Here we are concerned with a better understanding of the transport processes through fibrous acoustic materials in order to predict the acoustical properties of lightweight glass wool samples. The other purpose of this paper is to relate fiber structure to transport and sound absorption coefficients of such random fiber geometries by choosing the appropriate microstructural parameters and formulating a suitable multiscale framework.

Tomadakis and Robertson [22] were probably the first to provide systematic computations of transport parameters over a wide range of porosities for three classes of random fibrous media: the structures formed by cylindrical fibers distributed randomly in 1, 2, or 3 directions were considered. One directional (1-d) random fiber structures have their axes parallel to each other, with their traces randomly distributed on the normal plane. Two-directional (2-d) random fiber structures have their axes on planes parallel to each other, with their positions and orientations randomly distributed on these planes. Threedirectional (3-d) random fiber structures have their axes randomly positioned and oriented in the three-dimensional space. Their results were however restricted to fiber models with equal diameters and lengths, and to limited data points in their investigations of the fiber orientation effect.

Using data on the microstructure of a non-woven acquired from two-dimensional reflected light microscope images of microsections of the non-woven, Schladitz et al. [15] constructed a model of a random fibrous media. The geometric model of a random fibrous media was using fiber thicknesses, porosity, and anisotropy of the fiber system as input parameters. The authors indicated that permeability simulations for the case where a radii distribution 
is used and in the other case where only the mean radius is considered (calculated in terms of number of fibers) lead to significantly different results (Fig. 7) [15], but they did not consider and describe the effect of fiber length. Moreoever, the permeability computations were not compared with experimental data, which does not allow one to conclude on the importance of fiber length for the quantitative modeling of permeability and sound absorption in random fibrous media.

Jensen and Raspet [25] numerically determined the thermoacoustic properties of fibrous materials from realizations of the geometric model pioneered by Schladitz et al. [15] using the hybrid thermal Lattice Boltzmann model [26]. Their objective was to test some extensions of the pore-based microstructural models of Attenborough [27] and Zwicker and Kosten [28] and of the relaxation model proposed by Wilson [29] against numerical data. They noted that both of these extended models yield a reliable prediction of thermoacoustic performance provided that the parameters of the models (shape factors and relaxation times) are selected to best fit the data. However, no correlations were given between the parameters of the fiber geometry and the results of the simulation data (acoustic parameters).

Peyrega et al. [30] presented fibrous detailed morphological characterization of a material made of wooden fibers by studying three-dimensional images acquired with an X-ray microtomograph with mathematical morphology. Because this mathematical characterization highlights the transverse isotropy of fiber orientation, Peyrega and Jeulin [16] modeled the studied sample by using a simple two-dimensional periodic array of circular cylinders. A stochastic model composed of overlapping discs was also presented by these authors based on a random Poisson point process. The porosity and the fiber radius of the models were determined according to the morphological characterization, using the measured porosity and a volume weighted average radius as input parameters. The fully coupled visco-thermal equations were solved both in the deterministic and stochastic models, and the predicted sound absorption found in quantitative agreement with measurements. In practice, the selection of the aforementioned average radius yields satisfactory results whenever the wave propagates perpendicularly to the plane to which all fibers belong.

This work follows a series of recent contributions by some of the authors [17, 23, 24], focusing on the determination of the acoustic properties of random fibrous materials from their microstructures. In these papers, numerical simulations of fluid flow through fibrous media were fed with microstructural descriptors of increased complexity, inferred from experiments. 
More precisely:

1. In [17], a reconstruction methodology of a random fibrous medium was proposed and validated using macroscopic measurements on the permeability and sound absorption. This first approach specifically accounts for the porosity of the material, as well as for fiber diameters and orientations, but does not involve a description of the fiber length.

2. The second contribution [24] built on the model developed in (1) and focused on numerically characterizing the sensitivity of the transport properties with respect to the microstructural parameters under consideration (namely, the porosity and fiber diameter and orientation).

3. In [23], the definition as to how "equivalent" microstructures (in terms of macroscopic properties) can be defined was addressed in a computational setting. A fibrous medium exhibiting a tight distribution of fiber diameters was purposely considered, and the effect of fiber length distribution was not studied.

The present effort aims to extend the above framework by addressing the case of a random network exhibiting high levels of statistical fluctuations for both the diameter and length of the fibers (it should be noticed at this point that the determination of the elastic properties of random fibrous media from their microstructural characteristics is out of the scope of this paper). Here, glass wool is considered as a prototypical material combining unique challenging features [31-33], such as wide distributions of fiber diameters, lengths, and angular orientations.

This paper is organized as follows. Sec. II is devoted to the experimental characterization of the acoustical fibrous materials under study, at both micro- and macro- scales. Sec. III deals with the definition of two local geometry models (a deterministic one and a stochastic one) that are fed into a multiscale framework to compute the transport properties and sound absorption coefficients. An experimental validation is provided throughout Sec. IV.

\section{EXPERIMENTAL CHARACTERIZATION OF THE RANDOM FIBROUS NETWORK AND TRANSPORT PROPERTIES}

This section is concerned with the statistical characterization of the fibrous network associated with manufactured glass wools. The raw materials and the experimental setup 
are first described in Sec. II A. The statistical results are then presented in Sec. II B.

\section{A. Description of the raw materials and methodological aspects}

In this study, we consider glass wools manufactured using sand, limestone and soda ash, as well as recycled off-cuts as the basic raw materials. The raw materials are melted in a furnace at very high temperatures, typically between $1,050{ }^{\circ} \mathrm{C}$ to $1,500{ }^{\circ} \mathrm{C}$. The mixture is drawn through tiny holes in rapidly rotating spinners. This process shapes it into fibers. The structure and density of the product may be adapted according to its final usage. Small quantities of binding agents are added to the fibers. The glass wool is then hardened in a curing oven at around $200{ }^{\circ} \mathrm{C}$, and is cut to the required size and shape. Ten products corresponding to different surface densities and various processing parameters are considered and labeled from 1 to 10 . From a manufacturing perspective, the products $1-5$ constitute a first class of products (which will be referred to as Class 1 below) characterized by a given surface density $\mathcal{S}_{1}$ (which is left undefined for industrial confidentiality reasons) yielding a porosity of about 0.995 . Similarly, the products $6-10$ exhibit a surface density $\mathcal{S}_{2} \gg \mathcal{S}_{1}$ (with a porosity equal to 0.985 approximately), and represent a second class of raw materials (which is denoted by Class 2). Samples of the material under consideration are shown at both the microscopic and macroscopic scales in Fig. 1.

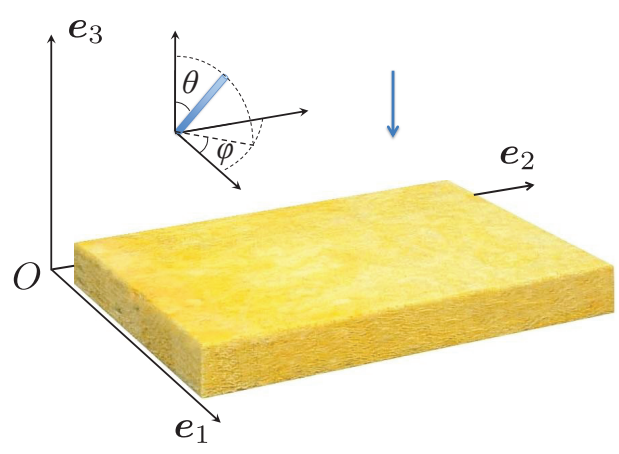

(a)

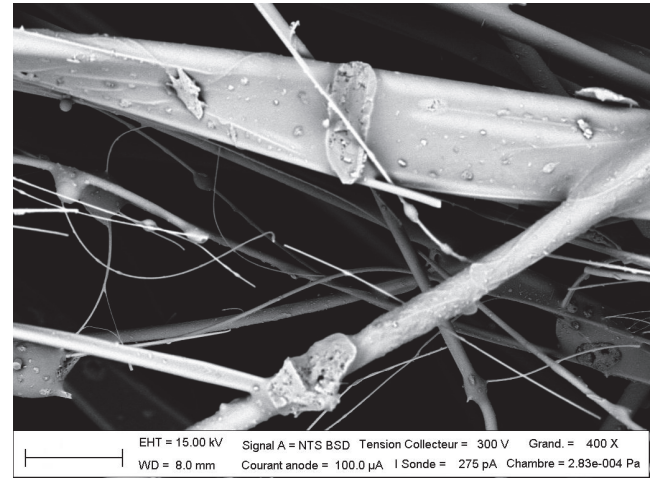

(b)

FIG. 1. (Color online) Macroscopic and microscopic views of a glass wool. (a) A pannel of fibrous material. The descendent arrow shows the airflow direction. Two angles $\theta$ and $\varphi$ are also defined in this coordinate system. (b) Associated microstructure obtained with a Scanning Electron Microscope (SEM) for the surface density $\mathcal{S}_{1}$. The heterogeneity of fiber diameters can easily be observed in the micrograph. The scale bar in the SEM image is $5 \times m_{D}$, where $m_{D}$ is the mean diameter of the analyzed fibers (see Eq. (28) for a detailed definition of $m_{D}$ ) 
In order to proceed with the multiscale analysis, the random fibrous network is characterized by measuring key geometrical parameters, including the diameters and lengths of the fibers, as well as their orientation angles in horizontal and vertical planes (the former are planes parallel to $\left(O, \boldsymbol{e}_{1}, \boldsymbol{e}_{2}\right)$, while the latter correspond to planes parallel to $\left(O, \boldsymbol{e}_{2}, \boldsymbol{e}_{3}\right)$ in the coordinate system depicted in Fig. 1). More specifically, the measures for the diameters and lengths are automatically obtained by using a granulomorphometer. The fibers are taken by coring with a syringe, the maximum length of the fibers being equal to or less than $3.6 \mathrm{~mm}$. Samples are blown up in order to ensure that the fibers are well separated. Indeed, dispersion of fibers is required because superposed fibers and bundles of fibers are not characterized. The fibers whose length is not more than three times the diameter are excluded from the analysis as well as the fibers emerging from the observation window of 2 $\mathrm{mm}$. It should be noticed that this $3: 1$ aspect ratio corresponds to the common definition of a fiber, and that a compromise must be found between the resolution and the field of view. Micrographs are then sequentially extracted and processed by an image recognition algorithm. The number of fibers characterized by this approach can be found in Tab. I.

\begin{tabular}{|c|c|c|c|c|c|c|c|c|c|c|}
\hline Product & 1 & 2 & 3 & 4 & 5 & 6 & 7 & 8 & 9 & 10 \\
\hline Number of fibers & 7377 & 9143 & 4662 & 3715 & 10451 & 6414 & 4719 & 6510 & 5981 & 17398 \\
\hline
\end{tabular}

TABLE I. This table shows the number of fibers that were characterized using the granulomorphometer for each product.

The horizontal and vertical orientations of the fibers are directly measured, inside a given plane, from SEM pictures. Here, it is assumed that the microstructure is reasonably homogeneous over the macroscopic domain, so that the spatial sampling does not introduce a bias. For each product, 40 pictures are extracted for each plane of interest at random locations (note that the horizontal plane corresponds to the surface of the panel of the fibrous material), as shown in Fig. 2.

On average, each product is characterized by 200 angular measurements in both the horizontal and vertical planes, using the ImageJ software [34]. Below, it is assumed that the thickness of the samples is small enough (typically less than $1 \mathrm{~mm}$ ) to make the effect of out-of-plane fibers negligible in the (two-dimensional) reconstruction of the microstructure. 


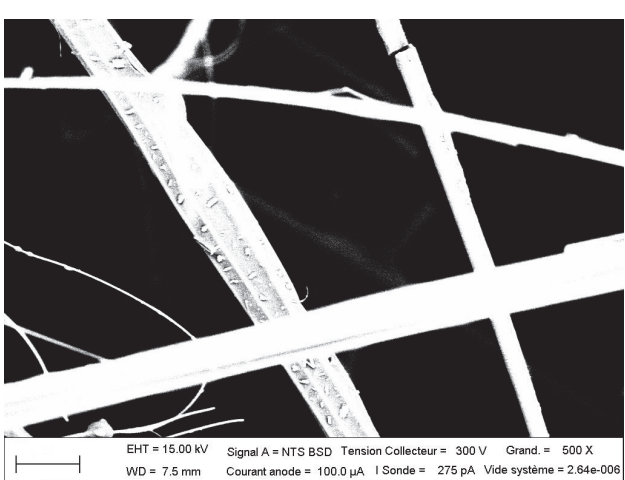

(a)

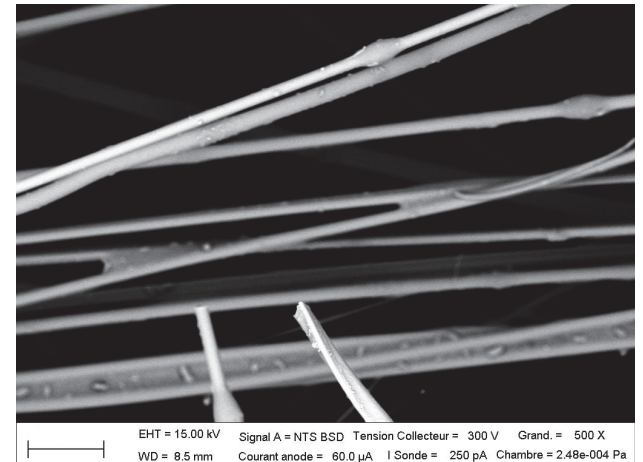

(b)

FIG. 2. Examples of micrographs. The scale bar in the SEM images is $5 \times m_{D}$, where $m_{D}$ is the mean diameter of the analyzed fibers (see Eq. (28) for a detailed definition of $m_{D}$ ). (a) Horizontal plane. Sample size is equal to $48.4 m_{D} \times 32.5 m_{D}$. (b) Vertical plane. Sample size is equal to $41.5 m_{D} \times 27.8 m_{D}$.

\section{B. Statistical characterization of the random microstructure}

From a statistical point of view, the probability density functions of interest are estimated through the nonparametric kernel method. The probability density functions associated with the fiber diameter and length, for product 7, are shown in Figs. 3 and 4, respectively. In these figures, the closest fits obtained with the Gamma probability density function are also reported. It should be noticed that the fiber lengths may be underestimated due to

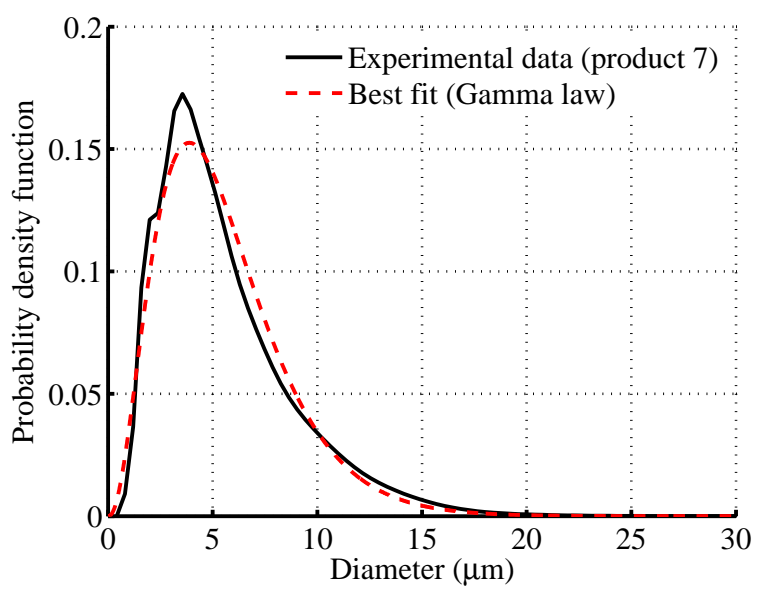

FIG. 3. (Color online) The estimated probability density function of fiber diameter is shown for product 7 .

the characterization strategy, as $(i)$ the extraction process, which is done using a syringe with a $3.6 \mathrm{~mm}$ catheter tip, breaks down over-lengthy fibers (beyond the $3.6 \mathrm{~mm}$ limit), 


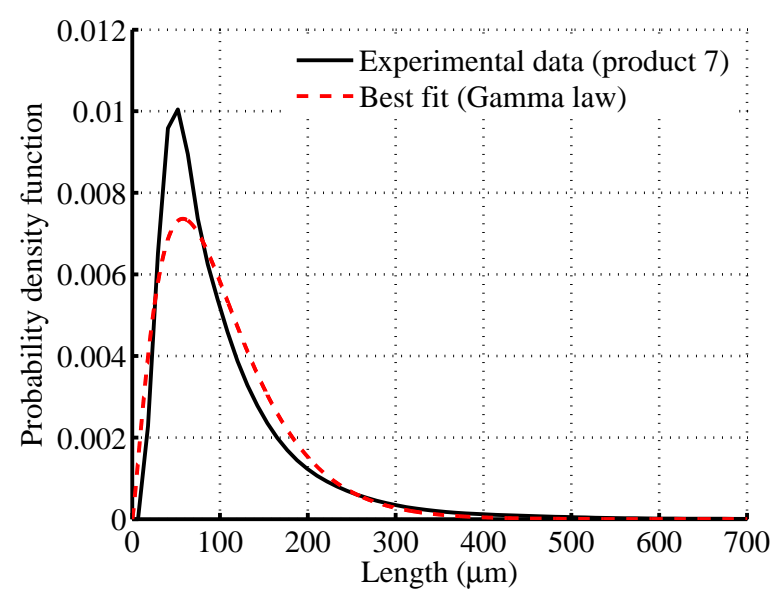

FIG. 4. (Color online) The estimated probability density function of fiber length is shown for product 7 .

and $(i i)$ objects that are not entirely contained in the field of view of the camera (during granulomorphometry) are not counted (approx. $2 \mathrm{~mm}$ ). For later use, we introduce the mean weighted diameter $D_{w}$, derived from the volume weighted diameter distribution [30] (in which each fiber has a weight equal to its volume) and defined as

$$
D_{w}=\frac{1}{\sum_{i=1}^{N_{f}} V_{i}} \sum_{i=1}^{N_{f}} V_{i} D_{i}
$$

where $V_{i}$ and $D_{i}$ are the volume and diameter associated with the $i$-th fiber (for product 7 , the mean value for the diameter estimated with the experimental data is equal to 5.52 $\mu \mathrm{m}$ and $\left.D_{w}=10.24 \mu \mathrm{m}\right)$. The probability density functions of the horizontal and vertical orientation angles are shown in Fig. 5 and 6, respectively. By convention, the support of the aforementioned probability density function is defined as $[0,180]$ (in [ $\left.{ }^{\circ}\right]$ ).

Based on these figures, the following modeling assumptions are formulated:

- the horizontal angle $\varphi$ follows a uniform distribution between 0 and $180\left[^{\circ}\right]$;

- the probability density function of the vertical angle $\theta$ provides information that corresponds to a preferred out-of-plane orientation generated by the manufacturing technique (which will be characterized by $\gamma$ hereinafter);

- the fiber diameter is distributed according to a Gamma distribution with shape parameter $1 / \delta_{D}^{2}$ and scale parameter $m_{D} \delta_{D}^{2}$, where $m_{D}$ and $\delta_{D}$ are the mean and coefficient 


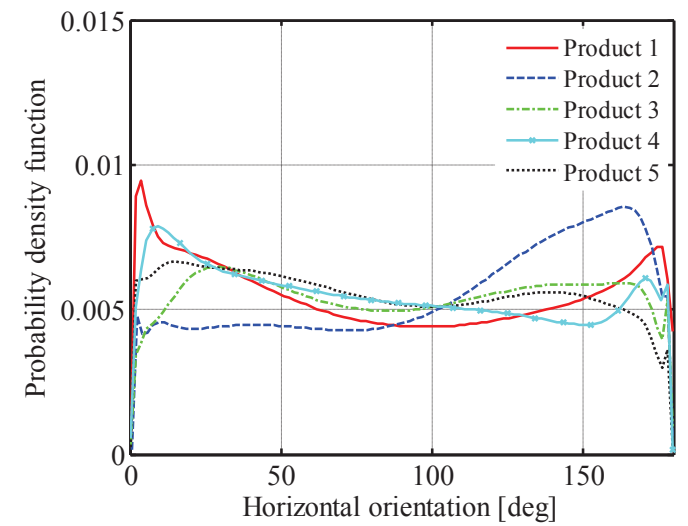

(a)

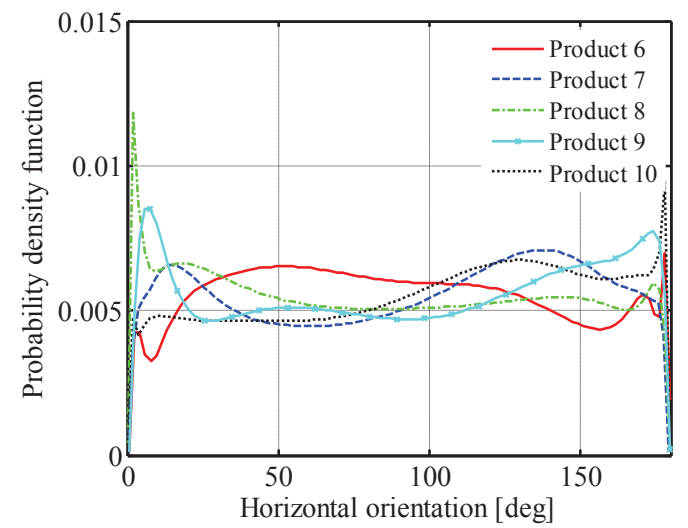

(b)

FIG. 5. (Color online) The estimated probability density function of the horizontal orientation angle $\varphi$ is shown for (a) Class 1 and (b) Class 2 .

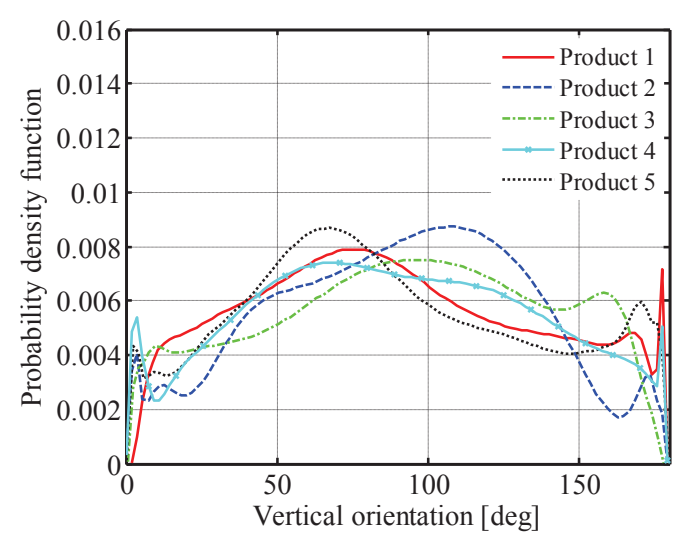

(a)

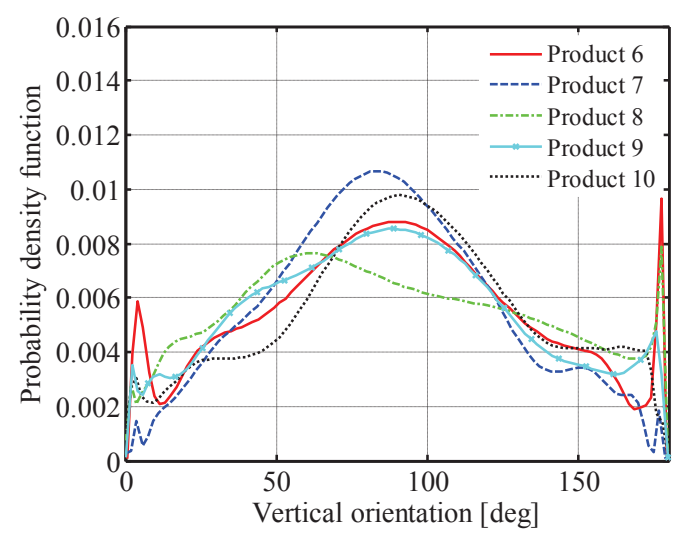

(b)

FIG. 6. (Color online) The estimated probability density function of the vertical orientation angle $\theta$ is shown for (a) Class 1 and (b) Class 2.

of variation of the diameter, respectively.

These assumptions, which are consistent with the results provided in [17], will be used in Sec. III in order to define the geometry of the fibrous media within the multiscale framework.

\section{Experimental characterization of transport properties}

The macroscopic density $\rho$, the porosity $\phi$ and the through-thickness permeability $K_{33}$ (along $\boldsymbol{e}_{3}$ ) are determined from direct measurements $[35,36]$. Regarding porosity measurements, we used a simple mass method to measure the open porosity, with large panels of fibrous materials $(600 \mathrm{~mm} \times 600 \mathrm{~mm})$ and a balance with a readability of $0.01 \mathrm{~g}$. The 
length and width of each panel was measured with a graduated steel rule, and the thickness was determined following EN823 standard method with a pin thickness gauge in five points and a plate pressure of $50 \mathrm{~Pa}$. The porosity $\phi$ was simply determined by $\phi=1-\rho_{1} / \rho_{m}$, in which $\rho_{1}$ is the macroscopic density (bulk density) of the panel of fibrous material deduced from its mass and dimensions and $\rho_{m}$ is the known density of the raw material $\left(2542 \mathrm{~kg} / \mathrm{m}^{3}\right.$ for the glass). This estimate assumes that the presence of the binder can be neglected, whose mass represents typically $5-10 \%$ of the product and whose density is not the one of the glass. A geometrical estimate of the thermal characteristic length $\Lambda^{\prime}$ can also be obtained from the knowledge of the porosity $\phi$ and the specific surface area $S_{p}$, since $S_{p}$ is a direct output of the granulomorphometry analysis (see Eq. (7)). The through-thickness permeability of each sample was measured for various static airflows with incremental reduction from 165 to 95 cubic centimeters/minute and determined for a linear airflow velocity of $0.5 \times 10^{-3} \mathrm{~m} / \mathrm{s}$ by graphical averaging or extrapolation to this value (ISO 9053 [37]). The tortuosity $\alpha_{\infty}$, the viscous characteristic length $\Lambda$, the static thermal permeability $k^{\prime}$, and the thermal characteristic lengths $\Lambda^{\prime}$ are determined using the indirect characterization method proposed in $[38,39]$. This requires, in particular, the measurement of both the equivalent dynamic bulk modulus and dynamic density, which is performed here by using the 3-microphone impedance tube method (see [40] for methodological aspects, as well as [41] for an application to foams and fibrous materials). In this study, the tube used for the measurements has a $40 \mathrm{~mm}$ inner diameter and the loudspeaker at one end generates a broadband random signal in the frequency band 200-4,000 Hz. It should be mentioned that the limp assumption [42] was used during the characterization process. The determination of the aforementioned parameters is considered satisfactory when both the measured equivalent dynamic bulk modulus and equivalent dynamic density of the materials are correctly characterized $[38,39]$.

\section{MULTI-SCALE ANALYSIS AND EXPERIMENTAL VALIDATION}

\section{A. Overview of the modeling methodology}

Let $\Omega$ be the representative volume element (RVE) under consideration (with boundary $\partial \Omega$ ), and let $\Omega_{f}$ denote the part of $\Omega$ occupied by the fluid phase (the viscosity of which 
is denoted by $\eta$ ). Let $\partial \Omega_{f}$ be the contact surface between the fluid and solid phases. We further introduce the following notations.

\begin{tabular}{|c|c|}
\hline Symbol & Property \\
\hline$\phi$ & Porosity \\
{$[K]$} & Static viscous permeability \\
$k^{\prime}$ & Static thermal permeability \\
$\alpha_{\infty}$ & High-frequency tortuosity \\
$\Lambda^{\prime}$ & Thermal characteristic length \\
\hline
\end{tabular}

TABLE II. List of symbols.

A four-step methodology is proposed as follows.

1. In the first step, a deterministic microstructure defined on a normalized domain $\Omega_{0}$ (with a unit characteristic length $L_{0}$ ) and exhibiting some representative morphological features of the random fibrous network (preferred out-of-plane orientation, mean weighted diameter) is introduced. This equivalent microstructure allows, on the one hand, to substantially reduce to computational cost associated with the multiscale simulations (when compared to a fully stochastic microstructural model). On the other hand, it enables the prediction of some transport properties, such as the static viscous permeability, on larger domains defined through a homothetic transformation of $\Omega_{0}$, at no additional cost. At this point, computational homogenization techniques are subsequently used to construct a map between the key microstructural parameters and the transport properties (see Secs. III B 1, III B 2 and III B 3).

2. In the second step (see Sec. III B 4) the above results are used to identify the length of the physical domain $\Omega$ preserving the macroscopic porosity, at a given and constant fiber diameter defined, for each product under consideration, by $D_{w}$ (see Eq. (1)). The transport properties of interest are then deduced either by appropriate scaling rules (as discussed in [43], for instance), or through compensation rules involving, in particular, some statistical properties estimated from the experimental database.

3. In the third step, detailed in Sec. III B 5, a fully stochastic microstructural model is introduced to assess the relevance of the equivalent microstructure developed in Step 1. This model is constructed using the empirical distributions for the microstructural parameters, and the impact of the modeling assumption for the fiber diameter is specifically explored. 
4. In the fourth step (see Sec. III B 6), acoustical models are introduced to define the acoustic properties of the fibrous media in terms of the macroscopic parameters determined from the computational homogenization framework.

These steps are detailed in the following sections.

\section{B. Definition of the microstructure and multiscale simulations}

\section{Definition of the equivalent microstructure}

Before setting up the multiscale framework, let us introduce the equivalent microstructure. The latter is a periodic model based on an ordered network of cylindrical fibers within a cubical unit cell incorporating measured morphological characteristics of the real fibrous material. Let $\Omega_{0}=([0,1])^{3}$ be the geometrical domain under consideration. Throughout this section, the superscript "(0)" refers to quantities of interest that are associated with $\Omega_{0}$. The fibrous network is then defined by inserting a set of $N$ traverse fibers with constant diameter $D_{0}$ such that the configuration comprises all possible combinations of horizontal and vertical angles defined by the probability mass functions

$$
p_{\varphi}(x)=\frac{1}{N_{\varphi}} \sum_{i=1}^{N_{\varphi}} \delta\left(x-\varphi^{(i)}\right)
$$

and

$$
p_{\theta}(x)=\frac{1}{N_{\theta}} \sum_{j}^{N_{\theta}} \delta\left(x-\theta^{(j)}\right),
$$

where $\delta$ is the Dirac measure at the origin (with $N=N_{\varphi} \times N_{\theta}$ ). In practice, the values of $N_{\varphi}$ and $N_{\theta}$ must be chosen to ensure both data consistency and macroscopic isotropy. Here, we set $N_{\varphi}=4$ and $N_{\theta}=2$, and the atoms defining the above probability mass functions [44] are selected as

$$
\varphi^{(i)}=(i-1) \frac{\pi}{4}, \quad \theta^{(j)}=\frac{\pi}{2}+(-1)^{j} \gamma
$$

where $\gamma$ is the angle introduced in Sec. II B and defined by

$$
\gamma=\mathbb{E}\left\{\left|\theta-\frac{\pi}{2}\right|\right\}
$$


The geometry hence obtained is schematically depicted in Fig. 7. It should be noticed that
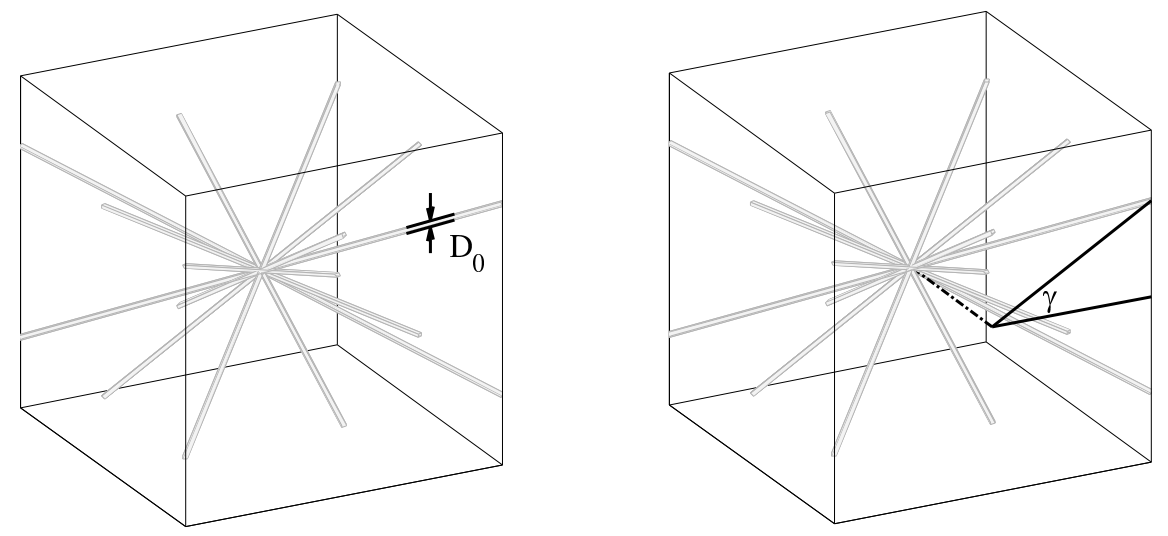

FIG. 7. Illustration of the proposed equivalent microstructure, parameterized by $D_{0}$ and $\gamma$.

the fibers are all interconnected at the center of the cell, and that the microstructure thus defined is $\Omega$-periodic.

\section{Definition of the porosity and thermal characteristic length}

The porosity $\phi$ and the thermal characteristic length $\Lambda^{\prime}$ are purely geometrical quantities that can be readily deduced from the definition of the microstructure. More precisely, the porosity is defined as

$$
\phi=\frac{V_{f}}{V_{T}},
$$

where $V_{f}=\left|\Omega_{f}\right|$ is the volume occupied by the fluid (air) and $V_{T}=|\Omega|$ is the total volume of the domain under consideration. The thermal characteristic length $\Lambda^{\prime}$ is given by

$$
\Lambda^{\prime}=\frac{2 \phi}{S_{p}}=\frac{2 V_{f}}{S_{f s}}
$$

in which $S_{f s}$ is the contact area between the fluid and the solid, and $S_{p}=S_{f s} / V_{T}$ denotes the specific surface area. The evolution of $\phi$ and $\Lambda^{\prime}$ (for the normalized domain $\Omega_{0}$ ) as a function of both the preferred out-of-plane orientation $\gamma$ and the fiber diameter $D_{0}$ is shown in Fig. 8. As expected, it is seen that the porosity $\phi$ is monotonically decreasing with $D_{0}$, regardless of the angle $\gamma$. Note that the drop in porosity does depend on $\gamma$, and that a decrease of $4 \%$ is observed for $\gamma=45^{\circ}$ [see Fig. 8(a)]. We note that this effect is reduced for smaller angles (which generate smaller fiber lengths, by construction). Moreover, increasing 


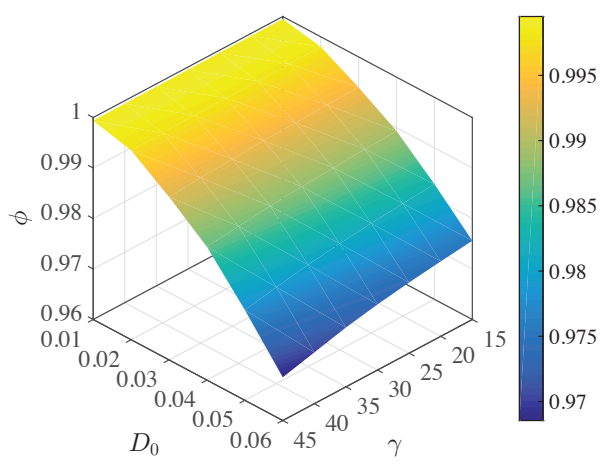

(a)

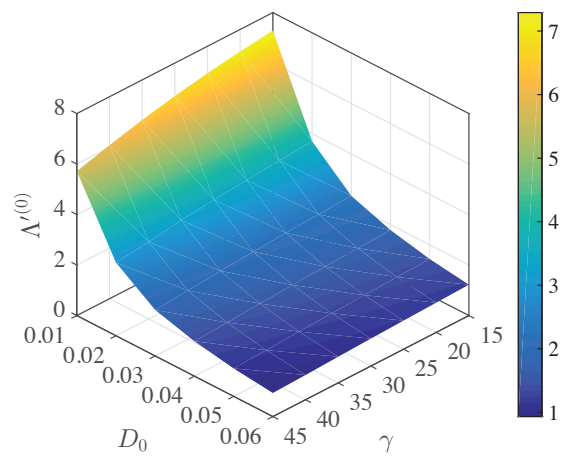

(b)

FIG. 8. (Color online) This figure shows the graphs of (a) the macroscopic porosity $\phi$ and (b) the thermal characteristic length $\Lambda^{\prime(0)}$ associated with $\Omega_{0}$, as a function of $D_{0}[-]$ and $\gamma\left[^{\circ}\right]$.

$\gamma$ for a given value of $D_{0}$ results in both a decrease of the fluid volume $V_{f}$ and an increase of the contact area $S_{f s}$, hence implying a decrease of $\Lambda^{\prime}$ in view of Eq. 7 [see Fig. 8(b)].

\section{Computational homogenization}

In this section, computational homogenization techniques are used to estimate the transport properties as a function of the parameters $D_{0}$ and $\gamma$ (see [45] for a survey). First of all, multiscale predictions of the static viscous permeability tensor are obtained. For this purpose, recall that the velocity $\boldsymbol{v}$ and the pressure $p$ of the (incompressible) fluid satisfy the momentum equation

$$
\eta \Delta \boldsymbol{v}-\nabla p=-\boldsymbol{G}
$$

and the mass conservation equation

$$
\nabla \cdot v=0
$$

in $\Omega_{f}$, where $\boldsymbol{G}$ is a source term and the symbol "." denotes the classical inner product in $\mathbb{R}^{3}$. The above system of Stokes equations is supplemented with the boundary condition

$$
v=0
$$


on $\partial \Omega_{f}$, with $(\boldsymbol{v}, p)$ periodic on $\partial \Omega$. The components of the static viscous permeability tensor $[K]$ can then be calculated as

$$
[K]_{i j}=\phi\left\langle\left[K^{*}\right]_{i j}\right\rangle, \quad 1 \leqslant i \leqslant j \leqslant 3
$$

where $\langle\cdot\rangle$ denotes volume averaging in the fluid phase, viz.

$$
\langle\cdot\rangle=\frac{1}{\left|\Omega_{f}\right|} \int_{\Omega_{f}} \cdot d V,
$$

and $\left[K^{*}\right]$ is such that

$$
v_{i}=-\frac{\left[K^{*}\right]_{i j}}{\eta} G_{j}
$$

Another quantity of interest is the static viscous tortuosity tensor $\left[\alpha_{0}\right]$ which reflects the dispersion of microscopic velocities $\boldsymbol{v}$ around their macroscopic average $\langle\boldsymbol{v}\rangle$,

$$
\left[\alpha_{0}\right]_{i j}=\frac{\phi\left\langle\left[K^{*}\right]_{p i}\left[K^{*}\right]_{p j}\right\rangle}{\left\langle\left[K^{*}\right]_{i i}\right\rangle\left\langle\left[K^{*}\right]_{j j}\right\rangle}
$$

In practice, three independent problems must be solved for defining the entries of $\left[K^{*}\right]$, each problem being defined by considering the source term $\boldsymbol{G}^{(i)}, 1 \leqslant i \leqslant 3$, such that $G_{j}^{(i)}=\delta_{i j}, 1 \leqslant j \leqslant 3$, with $\delta_{i j}$ the Kronecker symbol $\left(\delta_{i j}=1\right.$ if $i=j, 0$ otherwise). In this work, the Stokes equations are solved by the finite element method. More specifically, 5-node MINI tetrahedral elements are used for the velocity field, while 4-node tetrahedral elements are used for the pressure field [46]. This $P^{1}$-bubble $/ P^{1}$ formulation satisfies the Babuška-Brezzi condition [47], and was implemented within in-house Matlab routines. The code verification was carried out by addressing various benchmarks proposed elsewhere in the literature (such as [48]). In order to ensure a proper refinement near the boundary layers, the mesh generation was performed by using the commercial software COMSOL Multiphysics. For $D_{0}=0.04 L_{0}[-]$ and $\gamma=15\left[^{\circ}\right]$, the finite element mesh contains 113887 elements (with a nonuniform density) and is shown in Fig. 9.

Since only through-thickness experiments are conducted, we restrict our attention below to the through-thickness components of tensorial macroscopic properties. We then denote by $K_{0}^{(0)}$ the effective scalar static permeability along $\boldsymbol{e}_{3}$, and the graph of the mapping $\left(D_{0}, \gamma\right) \mapsto K_{0}^{(0)}$ is shown in Fig. 10. The static viscous permeability $K_{0}^{(0)}$ decreases as the 


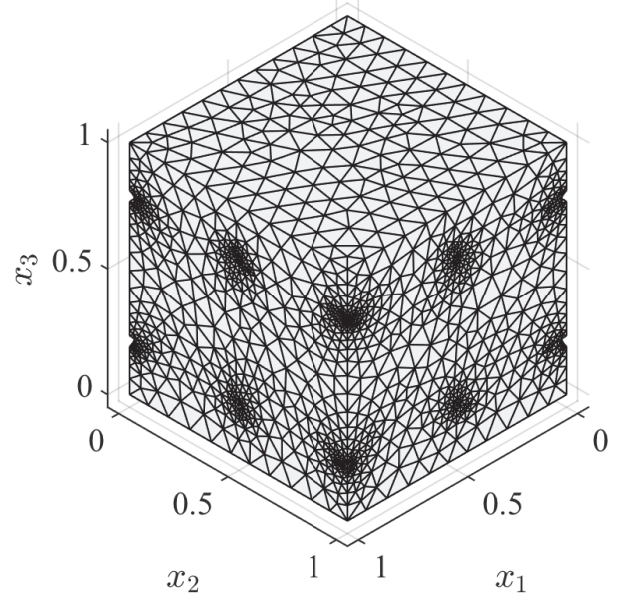

FIG. 9. Meshed view of the equivalent microstructure.

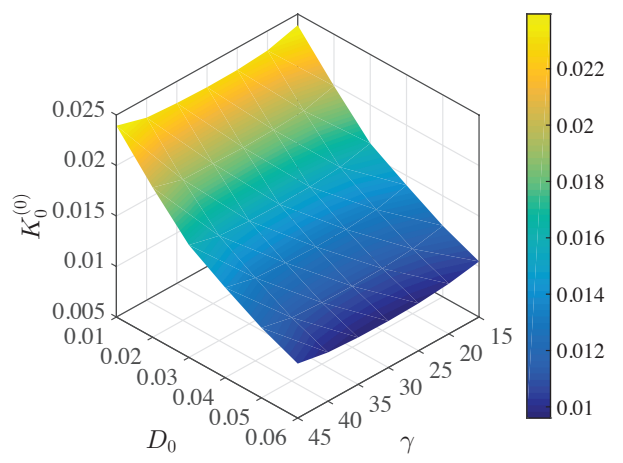

(a)

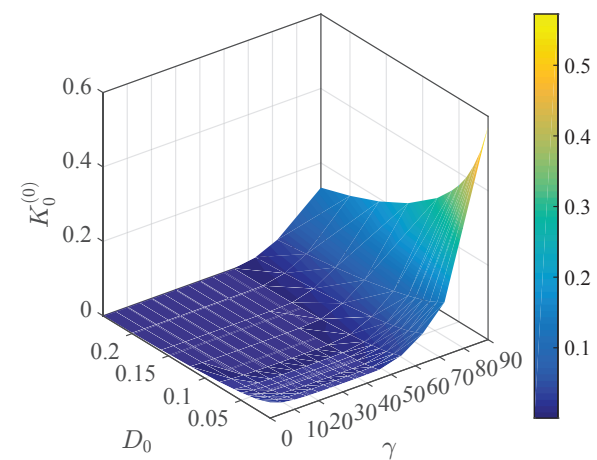

(b)

FIG. 10. (Color online) These figures show the graphs of the effective permeability $K_{0}^{(0)}$ associated with $\Omega_{0}$, as a function of $D_{0}[-]$ and $\gamma\left[{ }^{\circ}\right]$. The range of angular values corresponds to (a) $15^{\circ} \leq$ $\gamma \leq 45^{\circ}$, and to (b) $0^{\circ} \leq \gamma \leq 90^{\circ}$.

fiber diameter increases (which corresponds to an increase in the specific airflow resistance). In the range of angles $15^{\circ} \leqslant \gamma \leqslant 45^{\circ}$, the effect of the microstructural parameter $\gamma$ on $K_{0}^{(0)}$ is relatively small (as compared to the effect of diameter $D_{0}$ ). This effect is, however, more pronounced for larger values of $\gamma$, especially when $\gamma$ tends to $90^{\circ}$ - in which case the fibers are aligned with the direction of the macroscopic flow [Fig. 10(b)]. These trends are expected since $\gamma$ corresponds to a preferred out-of-plane orientation of fibers: as a result, drag forces controlling the amount of relative flow induced in the fluid-saturated porous material increase as $\gamma$ decreases.

The next step involves the estimation of the high-frequency tortuosity $\alpha_{\infty}$ and viscous characteristic length $\Lambda$. These quantities of interest can be obtained by solving the following 
potential equations

$$
\boldsymbol{E}=-\nabla \Phi+\boldsymbol{e}, \quad \boldsymbol{\nabla} \cdot \boldsymbol{E}=0
$$

in $\Omega_{f}$, where $\boldsymbol{E}$ is the electric field, $\Phi$ is an electric potential and $\boldsymbol{e}$ is a (given) input macroscopic electric field. The associated boundary conditions write

$$
\boldsymbol{E} \cdot \boldsymbol{n}=0
$$

on $\partial \Omega_{f}$, with $\boldsymbol{n}$ the unit vector normal to the boundary $\partial \Omega_{f}$, and $\Phi$ periodic on $\partial \Omega$. The through-thickness high-frequency tortuosity is then defined by considering the microscopic field $\boldsymbol{e}=\boldsymbol{e}_{3}$ and by letting

$$
\alpha_{\infty}=\frac{\langle\boldsymbol{E} \cdot \boldsymbol{E}\rangle}{\langle\boldsymbol{E}\rangle \cdot\langle\boldsymbol{E}\rangle}
$$

The associated viscous characteristic length reads as

$$
\Lambda=2\left(\int_{\Omega_{\mathrm{f}}} \boldsymbol{E} \cdot \boldsymbol{E} \mathrm{d} V\right)\left(\int_{\partial \Omega_{\mathrm{f}}} \boldsymbol{E} \cdot \boldsymbol{E} \mathrm{d} S\right)^{-1}
$$

Finally, the static thermal permeability ${k^{(0)}}^{(i s}$ estimated by solving the heat transfer equation, namely

$$
\Delta u=-1
$$

in $\Omega_{f}$, with

$$
u=0
$$

on $\partial \Omega_{f}$ and $u$ periodic on $\partial \Omega$. The transport parameter ${k^{\prime}}^{(0)}$ is then obtained as

$$
k^{(0)}=\langle u\rangle
$$

A thermal tortuosity parameter $\alpha_{0}^{\prime}$, that is a scalar analogous to the viscous tortuosity tensor $\left[\alpha_{0}\right]_{i j}$, can be defined as

$$
\alpha_{0}^{\prime}=\frac{\left\langle u^{2}\right\rangle}{\langle u\rangle^{2}}
$$

The two boundary value problems that are respectively defined by Eqs. (15-16) and Eqs. (19-20) are presently solved by using 4-node tetrahedral finite elements. The dependence of the high-frequency tortuosity and viscous characteristic length on parameters $D_{0}$ and $\gamma$ 
is illustrated below in Fig. 11. It is observed that the high-frequency tortuosity $\alpha_{\infty}^{(0)}$ remains

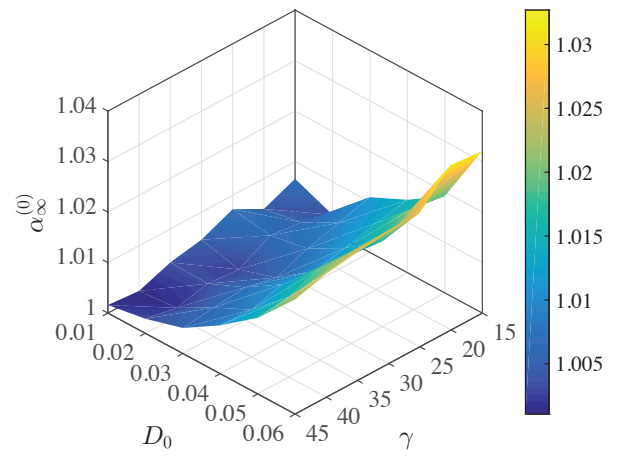

(a)

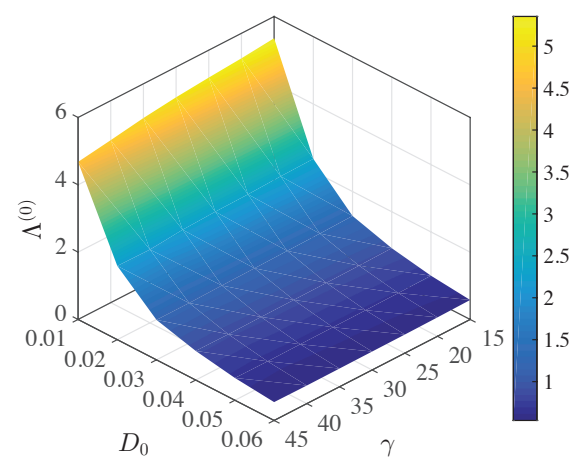

(b)

FIG. 11. This figure shows the graphs of (a) the high-frequency tortuosity $\alpha_{\infty}^{(0)}$ and (b) the viscous characteristic length $\Lambda^{(0)}$ associated with $\Omega_{0}$, as a function of $D_{0}[-]$ and $\gamma\left[^{\circ}\right]$.

very close to 1, with a range of variation in the order of magnitude of typical measuremen$\mathrm{t}$ uncertainties, and that its values increase together with the values of the diameter $D_{0}$. Moreover, when the angle $\gamma$ decreases, a small increase in $\alpha_{\infty}^{(0)}$ can be noticed. As illustrated in Fig. 11(b), the values of $\Lambda^{(0)}$ follow the same trend as the values of $\Lambda^{\prime(0)}$, with $\Lambda^{(0)} \leqslant \Lambda^{(0)}$. To compare the thermal $\Lambda^{\prime(0)}$ and viscous $\Lambda^{(0)}$ characteristic lengths further, we also present, in Fig. 12, the evolution of the ratio $\Lambda^{\prime(0)} / \Lambda^{(0)}$. For the angles under consideration, the above

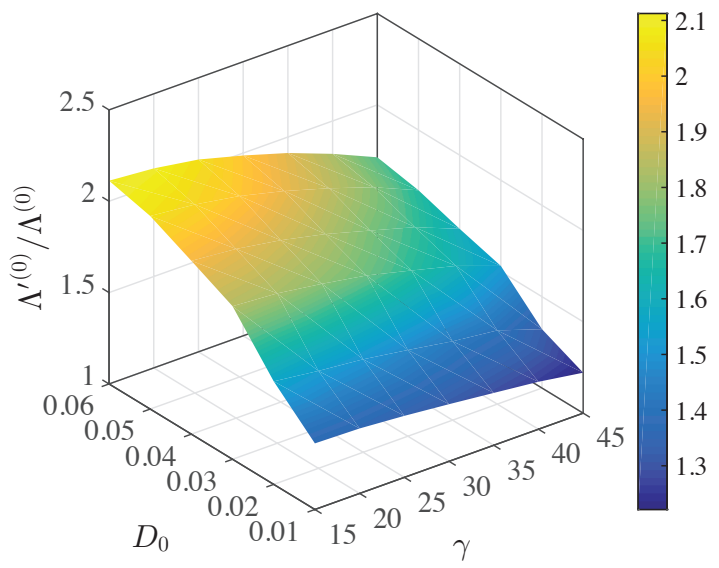

FIG. 12. (Color online) This figure shows the graph of the ratio $\Lambda^{\prime(0)} / \Lambda^{(0)}$ associated with $\Omega_{0}$, as a function of $D_{0}[-]$ and $\gamma\left[^{\circ}\right]$.

ratio decreases from 2.1 (which corresponds to the situation where the fibers are not entirely perpendicular to the air velocity direction, with $D_{0}=0.06$ ) to 1.2 (which corresponds to 
the angle $\gamma=45^{\circ}$, with a smaller diameter $D_{0}=0.01$ ). As expected, it is seen that the ratio $\Lambda^{\prime(0)} / \Lambda^{(0)}$ increases whenever the fibers are placed perpendicular to the air velocity direction or when the fiber diameter is sufficiently large (the same conclusion holds when these situations, which introduce constrictions in the fluid domain, are combined). Note that this effect appears as being more prominent when the angle $\gamma$ takes small values (in particular, one has $\Lambda^{\prime(0)} / \Lambda^{(0)} \approx 5$ for $\gamma=0^{\circ}$ and $\left.D_{0}=0.25\right)$. Finally, the graphs of the static thermal permeability $k^{(0)}$ and the ratio $k^{(0)} / K_{0}^{(0)}$ are displayed in Fig. 13. Obviously,

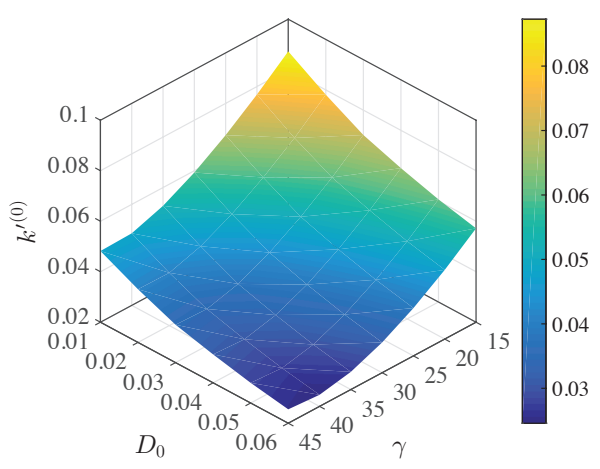

(a)

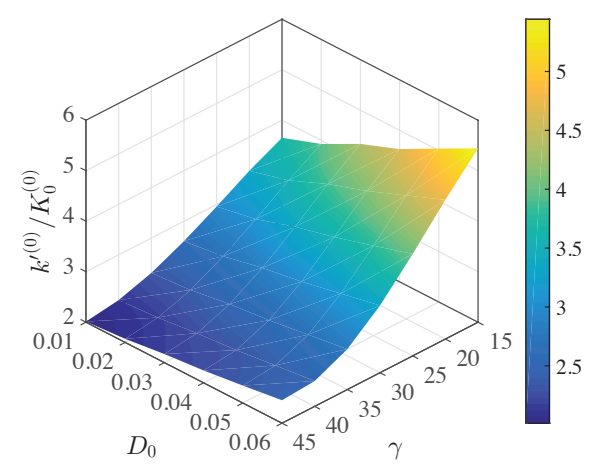

(b)

FIG. 13. (Color online) This figure shows the graphs of (a) the static thermal permeability $k^{(0)}$ and (b) the ratio ${k^{\prime}}^{(0)} / K_{0}^{(0)}$ associated with $\Omega_{0}$, as a function of $D_{0}[-]$ and $\gamma\left[^{\circ}\right.$.

the static thermal permeability increases when the diameter $D_{0}$ increases or when the angle $\gamma$ decreases. Because the thermal permeability ${k^{\prime}}^{(0)}$ is independent of the angle $\gamma$ at constant porosity (see [17]), the ratio $k^{(0)} / K_{0}^{(0)}$ is seen to increase as $K_{0}^{(0)}$ decreases (which corresponds to the case of decreasing angles).

\section{Definition of scaling rules}

In this section, we explore the definition of scaling rules. For a given quantity of interest (transport parameter), a scaling rule is defined as the correction to apply to the estimate obtained with the normalized unit cell $\Omega_{0}$ in order to deduce corresponding values for the (real) porous medium.

First of all, the parameters $\alpha_{\infty}, \alpha$ and $\alpha^{\prime}$ are independent of the domain size and are therefore directly extracted from the simulations that were carried out in Sec. III B 3.

Next, the effective characteristic length $L$ of domain $\Omega$ is determined, for each product, 
by imposing that

$$
\phi_{0}\left(D_{w} / L, \gamma^{e x p}\right)=\phi_{0}\left(D_{0} / L_{0}, \gamma^{e x p}\right)=\phi^{e x p}
$$

where $D_{w}$ was defined in Eq. (1), $L_{0}=1$ by construction and the superscript "exp" refers to experimental values. When a laboratory measurement of the porosity $\phi^{\exp }$ and the preferred out-of-plane orientation $\gamma^{e x p}$ are available, the unit cell aspect ratio $D_{0} / L_{0}$ can be identified using the second equality in Eq. (23). The first equation then yields an estimate of $L$, provided that $D_{w}$ is available through experiments, so that the equivalent microstructure of the fibrous media is completely defined. In practice, this characteristic length ranges from 200 to $400[\mu \mathrm{m}]$ in the numerical experiments, and this range falls within the $95 \%$ confidence interval estimated from the database. Moreover, it can be shown that static viscous and thermal permeability scale with the square of the characteristic length [43], namely

$$
[K]_{i j}=L^{2} K_{0}^{(0)} \delta_{i j}, \quad k^{\prime}=L^{2} k^{(0)}
$$

A first compensation rule for the viscous and thermal characteristic lengths may similarly be obtained as

$$
\Lambda=L \Lambda^{(0)}, \quad \Lambda^{\prime}=L \Lambda^{\prime(0)}
$$

The above definitions are unsatisfactory since these characteristic lengths $\Lambda$ and $\Lambda^{\prime}$ are related to the contact area $S_{f s}$ between the fluid and the solid, and therefore to the fiber diameters. In the proposed equivalent microstructure, all the fibers have the same value of diameter $D_{0}$. However, glass wools present a spread distribution of fiber diameters (Fig. 3). This aspect has not yet been taken into account in the definition of the equivalent microstructure. Therefore, in order to further elaborate on the adaptation of these quantities, let us consider the case of the thermal characteristic length $\Lambda^{\prime}$, and denote by $\Lambda_{c}^{\prime}$ the value that is obtained by taking into account the distributions for both the fiber length and diameter. By definition (see Eq. (7)), one has

$$
\frac{\Lambda_{c}^{\prime}}{\Lambda^{\prime}}=\frac{S_{f s}}{S_{f s}^{c}}
$$

where $S_{f s}^{c}$ is the contact area between the fluid and the solid in the configuration with fiberdependent diameters. Upon using the preservation of the macroscopic porosity, it can then 
be shown that

$$
\frac{\Lambda_{c}^{\prime}}{\Lambda^{\prime}}=\frac{\mathbb{E}\left\{D^{2}\right\}}{D_{w} \mathbb{E}\{D\}}
$$

Since the fiber diameter is assumed to follow a Gamma distribution, it follows that

$$
\mathbb{E}\{D\}=m_{D}
$$

and

$$
\mathbb{E}\left\{D^{2}\right\}=m_{D}^{2}\left(1+\delta_{D}^{2}\right)
$$

where $m_{D}$ and $\delta_{D}$ are the mean and coefficient of variation of the diameter $D$ (which can be estimated from the experimental data). Hence, a more accurate value of the thermal characteristic length can be obtained as

$$
\Lambda_{c}^{\prime}=C_{f} \Lambda^{\prime}
$$

where $C_{f}$ is a compensation factor given by

$$
C_{f}=\frac{m_{D}\left(1+\delta_{D}^{2}\right)}{D_{w}}
$$

It should be noticed that $C_{f}=1$ when the diameter remains constant for all fibers, and that no compensation factor was used to determine the viscous characteristic length $\Lambda$ (given that this quantity depends on the local field $\boldsymbol{E})$.

\section{Definition of a model for the random microstructure}

We now introduce a standard model for the true random microstructure. The purpose of this analysis is threefold: $(i)$ to clearly demonstrate the effect of the fiber length distribution; (ii) to verify whether or not retaining a mean value enables the simulation of polydisperse fibrous media; (iii) to study the impact of sample-to-sample randomness.

The random microstructure is parametrized, in part, by the angles defining the orientation of the fibers: these angles are sampled in accordance with the probability law inferred from the database (this is in contrast with the equivalent microstructure introduced in Sec. III B 1, for which fiber orientation remains deterministic). Regarding the definition of the diameters, 
three types of random microstructure are considered as follows:

- in the first random model, corresponding to Polydisperse Fibrous Media (PDFM), the diameter is randomly sampled for each fiber, according to the Gamma law estimated from the data (see Fig. 3 for instance);

- in the second model, corresponding to Polydisperse Fibrous Media with Weighted Diameters (PDFM-WD), the diameter of each fiber is weighted by its volume and the empirical distribution of the weighted diameters thus defined is sampled;

- in the third type of microstructure, the diameter is set equal to $D_{w}$ (Monodisperse Fibrous Media with Weighted Diameters (MDFM-WD)), regardless of the fiber under consideration.

Once the number of fibers has been determined, the sampling of the fiber diameter and length is performed iteratively to populate the RVE, using either a standard random generator (for labeled distributions) or the inverse transform method with empirical distributions (for the probability law involving weighted diameters). Similarly, the orientation angles are sampled according to their empirical distributions, and the center of the fibers is sampled according to a uniform distribution. Note that the fibers are allowed to intersect, since it does not affect the prediction of transport properties. In order to ensure the periodicity of the medium, fibers exiting the RVE are forced to re-enter the domain from the opposite side. The number of fibers hence generated for each product, together with the associated number of finite elements (FE) ensuring convergence, can be found in Tab. III .

\begin{tabular}{|c|c|c|}
\hline Product & Number of fibers & Number of FE \\
\hline 1 & 200 & 319160 \\
2 & 211 & 321900 \\
3 & 231 & 374567 \\
4 & 221 & 332850 \\
5 & 349 & 469598 \\
6 & 123 & 290172 \\
7 & 122 & 281427 \\
8 & 116 & 291316 \\
9 & 109 & 270661 \\
10 & 131 & 318144 \\
\hline
\end{tabular}

TABLE III. List of parameters related to the computational model for each product. 
One realization of the random microstructure with fiber-dependent diameters and the associated FE mesh are shown in Fig. 14 for product 1. The solution fields for one component

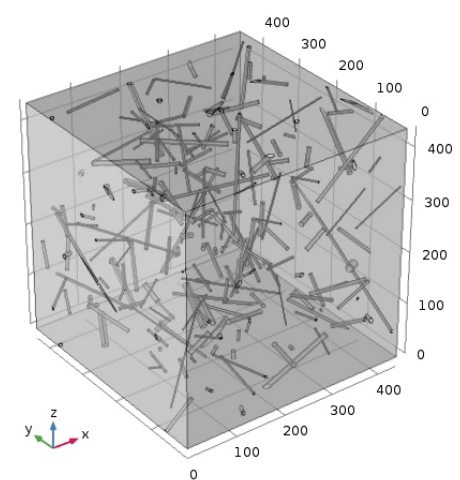

(a)

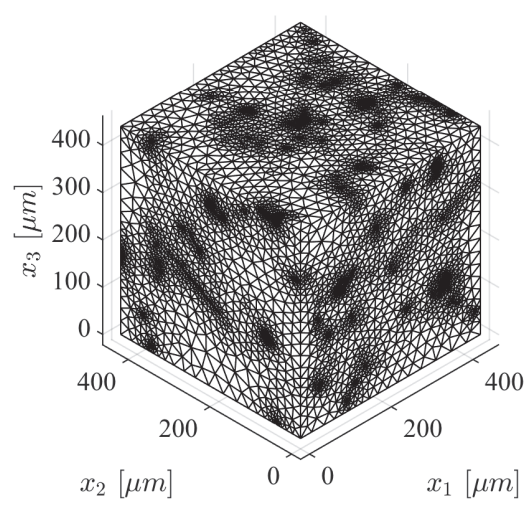

(b)

FIG. 14. One realization of the random microstructure associated with product 1 and the associated mesh view. (a) PDFM model of the random microstructure. (b) Corresponding mesh view.

of the velocity field and scaled concentration field are finally shown, for illustration purposes, in Fig. 15. It can be observed that the fluid-flow paths are more localized and follow a more tortuous path as compared to the heat diffusion field $\left(\alpha_{033}>\alpha_{0}^{\prime}\right)$.

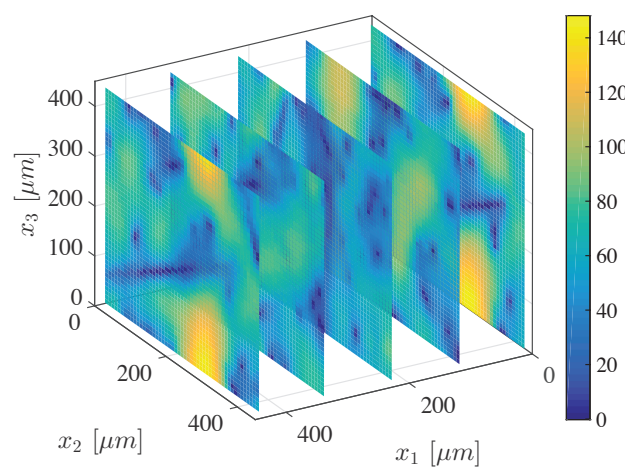

(a)

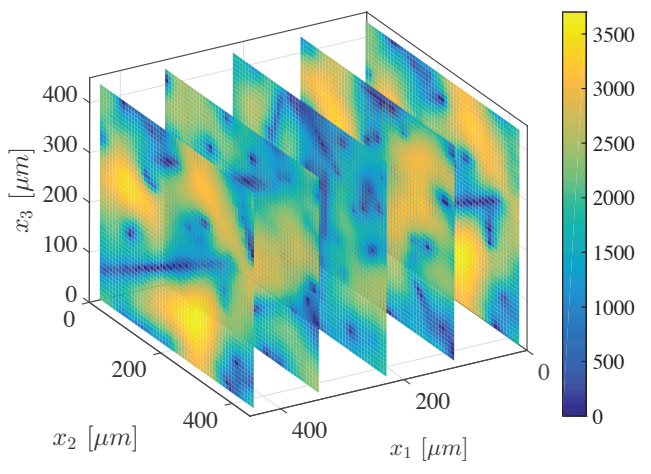

(b)

FIG. 15. (Color online) Solution fields associated with the realization of the random microstructure shown in Fig. 14. (a) Velocity field $\boldsymbol{x} \mapsto v_{3}(\boldsymbol{x})\left[\mu m . s^{-1}\right]$ associated with the source term $\boldsymbol{G}^{(3)}$. (b) Scaled concentration field $\boldsymbol{x} \mapsto u(\boldsymbol{x})\left[\mu \mathrm{m}^{2}\right]$. 


\section{Definition of the acoustic properties}

The effective density $\rho_{\text {eff }}(\omega)$ and the effective bulk modulus $K_{\text {eff }}(\omega)$ of the fluid phase can be evaluated by simple analytic admissible functions [10-13] as follows:

$$
\rho_{\mathrm{eff}}(\omega)=\rho_{0} \alpha_{\infty}\left[1+\frac{1}{\tilde{\omega}} f(\tilde{\omega})\right], \quad \frac{1}{K_{\mathrm{eff}}(\omega)}=\frac{1}{K_{a}}\left\{\gamma-(\gamma-1)\left[1+\frac{1}{j \tilde{\omega}^{\prime}} f^{\prime}\left(\tilde{\omega}^{\prime}\right)\right]^{-1}\right\}
$$

where $\rho_{0}$ is the air density at rest, $K_{a}$ is the adiabatic bulk modulus of air and $\gamma$ is its specific heat ratio. The quantities $\tilde{\omega}$ and $\tilde{\omega}^{\prime}$ are dimensionless viscous and thermal angular frequencies given by the following expressions:

$$
\tilde{\omega}=\frac{\omega}{\nu} \frac{K_{33} \alpha_{\infty}}{\phi}, \quad \tilde{\omega}^{\prime}=\frac{\omega}{\nu^{\prime}} \frac{k^{\prime}}{\phi}
$$

with $\nu^{\prime}=\nu / P_{r}, \nu$ the kinematic viscosity and $P_{r}$ the Prandtl number $\left(P_{r} \simeq 0.71\right.$ for air). Moreover, $f$ and $f^{\prime}$ are shape functions defined by

$$
f(\tilde{\omega})=1-P+P \sqrt{1+\frac{M}{2 P^{2}} j \tilde{\omega}}, \quad f^{\prime}\left(\tilde{\omega}^{\prime}\right)=1-P^{\prime}+P^{\prime} \sqrt{1+\frac{M^{\prime}}{2 P^{\prime 2}} j \tilde{\omega}^{\prime}}
$$

The quantities $M, M^{\prime}, P$ and $P^{\prime}$ are referred to as dimensionless shape factors and are given by

$$
M=\frac{8 K_{33} \alpha_{\infty}}{\Lambda^{2} \phi}, \quad M^{\prime}=\frac{8 k^{\prime}}{\Lambda^{\prime 2} \phi}, \quad P=\frac{M}{4\left(\frac{\alpha_{033}}{\alpha_{\infty}}-1\right)}, \quad P^{\prime}=\frac{M^{\prime}}{4\left(\alpha_{0}^{\prime}-1\right)} .
$$

. The complete model, corresponding to the refined "Johnson-Champoux-Allard-PrideLafarge" (JCAPL) model [10-13], relies on 8 parameters, namely $\left(\phi, K_{33}, k^{\prime}, \alpha_{\infty}, \Lambda, \Lambda^{\prime}\right.$, $\left.\alpha_{033}, \alpha_{0}^{\prime}\right)$. For $P=P^{\prime}=1$, the dynamic response functions reduce to a 6 -parameter model $\left(\phi, K_{33}, k^{\prime}, \alpha_{\infty}, \Lambda, \Lambda^{\prime}\right)$, known as the "Johnson-Champoux-Allard-Lafarge" (JCAL) or "Johnson-Lafarge" model $[10,11,13]$. Below, it is assumed that the frame of the porous material is limp (flexible), so that the effective density of the equivalent-fluid medium is defined as [42]:

$$
\rho_{\mathrm{eq}}(\omega)=\frac{\rho_{\mathrm{eff}}(\omega) m-\phi \rho_{0}^{2}}{\rho_{\mathrm{eff}}(\omega)+\phi\left(m-2 \rho_{0}\right)}
$$

where $m=\rho_{1}+\phi \rho_{0}$ is the total apparent mass of the equivalent fluid limp medium, and $\rho_{1}$ is the in vacuo bulk density of the medium. Let $K_{\text {eq }}(\omega)=K_{\text {eff }}(\omega) / \phi$ denote the bulk modulus 
of the so-called rigid-frame equivalent-fluid medium. Assuming plane wave solutions varying as $\exp \left[j\left(\omega t-q_{\mathrm{eq}}(\omega) x\right)\right]$, where $q_{\mathrm{eq}}(\omega)$ represents the wave number of the equivalent fluid medium, $\rho_{\text {eq }}(\omega)$ and $K_{\text {eq }}(\omega)$ can be used to calculate the wave number and the characteristic impedance of the equivalent fluid medium as:

$$
q_{\mathrm{eq}}(\omega)=\omega \sqrt{\frac{\rho_{\mathrm{eq}}(\omega)}{K_{\mathrm{eq}}(\omega)}}, \quad Z_{\mathrm{eq}}(\omega)=\sqrt{\rho_{\mathrm{eq}}(\omega) K_{\mathrm{eq}}(\omega)} .
$$

The sound absorption coefficient at normal incidence of a porous material layer of thickness $L_{s}$, backed by a rigid wall, is evaluated by

$$
\alpha=1-\left|\frac{Z_{n}-1}{Z_{n}+1}\right|^{2}
$$

where

$$
Z_{n}=-j \frac{Z_{\mathrm{eq}}}{Z_{0}} \cot \left(q_{\mathrm{eq}} L_{s}\right)
$$

is the effective normal impedance on the free face of the excited material, and $Z_{0}$ denotes the characteristic impedance of ambient air.

\section{EXPERIMENTAL VALIDATION}

In this section, the relevance of the framework is assessed by comparing the numerical predictions with experimental results on selected transport parameters (see Sec. IV A) and sound absorption coefficients (see Sec. IV B).

\section{A. Experimental validation on transport parameters}

The results are first presented for the static viscous permeability and viscous characteristic length in Fig. 16. While the PDFM model yields an underestimation of the static viscous permeability, it is seen that a quantitative appreciation of the latter can be obtained using either the equivalent microstructure (see Sec. IIIB1) or the random microstructure (see Sec. III B 5) with weighted diameters (PDFM-WD and MDFM-WD); see Fig. 16(a). Fur-

thermore, the results obtained for the PDFM-WD and MDFM-WD models remain pretty close to each other, suggesting that the MDFM-WD model may be more appealing from a 


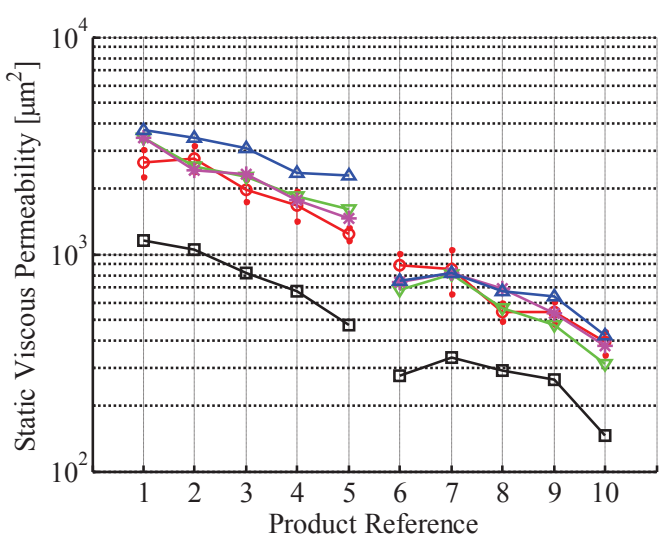

(a)

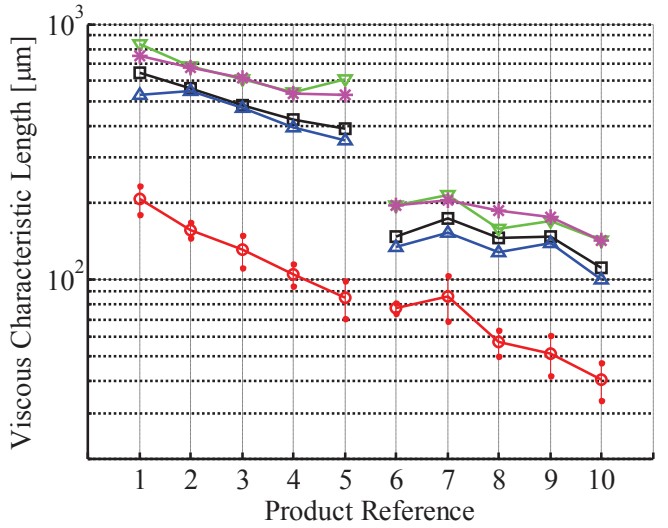

(b)

FIG. 16. (Color online) Comparison between numerical simulations and experimental data for the viscous characteristic dimensions. (a) Graph of the static viscous permeability. (b) Graph of the viscous characteristic length. Colors and symbols: experimental data (o, red); equivalent microstructure $(\triangle$, blue); random microstructure with diameters distributed according to a Gamma law - PDFM ( $\square$, black); random microstructure with weighted diameters - PDFM-WD ( $\nabla$, green); random microstructure with constant fiber diameter (equal to $\left.D_{w}\right)$ - MDFM-WD (*, magenta).

computational standpoint-since it does not require sampling the distribution for the diameters. A few comments regarding the relevance of considering weighted diameters in complex random fibrous webs are relevant at this point. If the distributions of the fiber parameters (diameter, length) are sharply peaked, the behavior of the overall system is similar to that of the individual elements. On the contrary, if these distributions are wide, the fibers with the largest relative volume (with respect to both diameter and length) dominate: in this case, the macroscopic permeability can be found by modeling a domain $\Omega$ of typical characteristic length $L$, in which fibers with weighted diameters are inserted.

Focusing next on Fig. 16(b), it is observed that the simulations overestimate the values of the viscous characteristic length $\Lambda$. For the random microstructure with weighted diameters (PDFM-WD), the ratio between the simulated and characterized data is, for instance, equal to $5.54 \pm 1.46$. In addition, it is seen that the calculations corresponding to the second class of products (Class 2), characterized by a higher surface density, are in better agreement with the measurements. This difference in ratio between the two groups of fibrous materials may be due to the high sensitivity of the numerical simulations with respect the number of inserted fibers (at a limited reconstructed volume)-as the solid volume fraction for the second class of fibrous material is three times higher than for the first class of fibrous materials $(1-\phi \approx 0.015$ for Class 2 , while $1-\phi \approx 0.005$ for Class 1$)$. 
Results related to the thermal characteristic length and the static thermal permeability are shown in Fig. 17. First of all, it should be noticed that the characterized values of

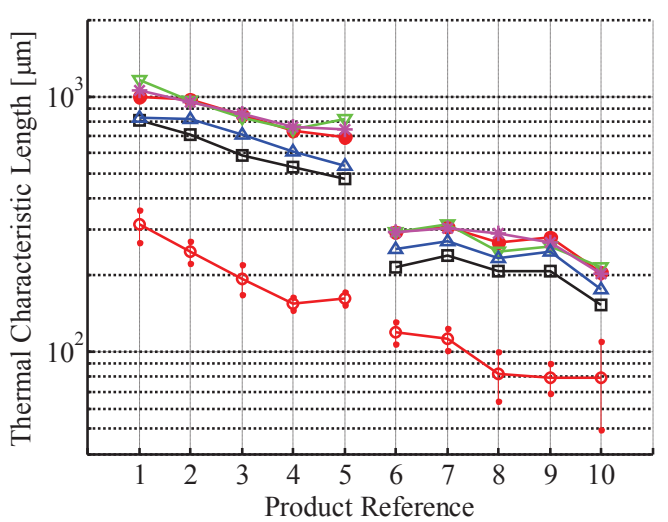

(a)

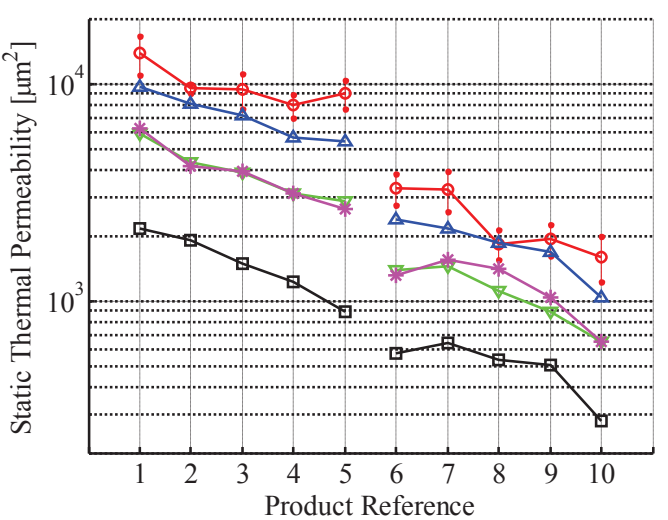

(b)

FIG. 17. (Color online) Comparison between numerical simulations and experimental data for the thermal characteristic dimensions. (a) Graph of the thermal characteristic length. (b) Graph of the static thermal permeability. Colors and symbols: experimental data with indirect characterization performed according to Ref. [39] (o, red); experimental data with direct geometrical characterization performed using the granulomorphometer $(\bullet$, red) (see Eq. 7); equivalent microstructure $(\triangle$, blue); random microstructure with diameters distributed according to a Gamma law - PDFM $(\square$, black); random microstructure with weighted diameters - PDFM-WD ( $\nabla$, green); random microstructure with constant fiber diameter (equal to $D_{w}$ ) - MDFM-WD (*, magenta).

the thermal characteristic length $\Lambda^{\prime}$ obtained from the acoustical and granulomorphometry method differ significantly (except for Reference 6), with a ratio of the properties measured by the two methods equal to $3.50 \pm 0.80$ for the ten products analyzed. This discrepancy can be explained by the aspect ratio limitation that was used in the granulomorphometry analysis, which excludes "objects" of potentially very small diameters. These objects play a significant role in decreasing $\Lambda^{\prime}$ by increasing $S_{f s}$ (see Eq. (7)), and this effect is expected to be captured more accurately by the acoustical method. Note that the variability of the results obtained by these two methods is quite significant for two products (References 7 and 10). Because we determined the thermal characteristic lengths from the granulomorphometry analysis, the values of this parameter are typically close to those obtained through the direct geometrical characterization method.

The simulated values of the static thermal permeability obtained with the equivalent microstructure are in good agreement with the experimental values, with a ratio between 
the simulated and experimental data equal to $0.75 \pm 0.12$ [see Fig. 17(b)]. While it can expected that the thermal interactions in the fiber webs (due to diffusion-controlled reactions) are more accurately described by a random microstructure mimicking more faithfully the geometrical data, the model PDFM-WD (random microstructure with weighted diameters) yields an underestimation of the static thermal permeability. For this particular case, the ratio between the experimental and simulated data is equal to $2.36 \pm 0.37$. Note that since all the data corresponding to the tortuosity were very close to one (and fall within the measurement uncertainty), they were not shown here.

It should be emphasized that most of the macroscopic parameters, while simulated without any adjusted parameters, are in accordance with the experimental results. Moreover, the models considered here all depend on the morphogranulometry that excludes long fibers from the analysis. The averaging procedure defined by Eq. (18) and involving the field $\boldsymbol{E}$ substantially favours smaller pores; in this discussion, the pore diameter at any point within the pore space is defined as the diameter of the largest sphere which contains this pore and remains wholly within the pore space [49]. The number of fibers that was characterized is considerably smaller than that from the sample size in the experiments. A likely explanation for our overestimate of the viscous characteristic length is that, in addition to the electric field carried in the main large channels through the pore space, a significant part of the electric field is being carried in parallel channels that are smaller than the simulated ones in the reconstructed fibrous samples. The contribution of these smaller channels to the electric field depends on their connectivity being properly described in the overall sample of glass wool. Fluid flow, on the other hand, is controlled by the largest connected channels $[50,51]$. Because our calculations were based on much smaller reconstructed volumes than the samples used for the experimental results determination, the limiting factor of our viscous characteristic length calculations appears to be the small sample sizes in the simulations. The same remark can be drawn for the $\Lambda^{\prime}$ parameter, whose determination is very sensitive to $\phi$ since $(1-\phi)$ is very small at low densities. 


\section{B. Experimental validation on sound absorption coefficients}

The predicted and characterized sound absorption coefficients at normal incidence for the geometrical models studied here are compared in Fig. 18. The characterization uses the JCAL model, while the simulation results are based on the full JCAPL model (both applied under the limp assumption). Tab. IV summarizes the sample thicknesses $L_{s}$ corresponding to each product. It is clear from these results that the experimental data are consistent with the weighted random model over the whole frequency range. It is interesting to see that the sound absorption of a glass wool can be predicted without adjustable parameters in the model for ten different products.

\begin{tabular}{|c|c|c|c|c|c|c|c|c|c|c|}
\hline Product & 1 & 2 & 3 & 4 & 5 & 6 & 7 & 8 & 9 & 10 \\
\hline$L_{s}(\mathrm{~mm})$ & 26.6 & 28.0 & 27.8 & 28.0 & 27.2 & 28.4 & 28.0 & 27.2 & 30.0 & 28.6 \\
\hline
\end{tabular}

TABLE IV. This table shows the sample thickness that was used when measuring sound absorption at normal incidence for each product.

Finally, it should be noticed that the above (samplewise) comparison is meaningful if and only if the condition of scale separation reasonably holds for all products under consideration. Here, the fulfilment of this condition was numerically checked through numerical experiments. From a methodological standpoint, the estimation of the transport properties was first carried out on a limited set of realizations of the random microstructure (for a given product). Given the small number of virtual samples (which is typically equal to 10), the coefficients of variation associated with the predicted properties were subsequently estimated by using the maximum likelihood method with appropriate labelled distributions (see Tab. $\mathrm{V}$ for the through-thickness permeability $K_{33}$, which is assumed at this point to follow a Gamma probability law; see [52] for a discussion). Except for product 10, the coefficient

\begin{tabular}{|c|c|c|c|c|c|c|c|c|c|c|}
\hline Product & 1 & 2 & 3 & 4 & 5 & 6 & 7 & 8 & 9 & 10 \\
\hline CV $([\%])$ & 3.8 & 4.9 & 3.8 & 5.5 & 2.9 & 5.6 & 4.7 & 4.7 & 5.4 & 7.9 \\
\hline
\end{tabular}

TABLE V. Estimated Coefficient of Variation $(\mathrm{CV})$ associated with $[K]_{33}$ for all products.

of variation is seen to be smaller or equal to $5 \%$, hence validating the assumption about scale separation (which can only be satisfied approximately in simulations). 
(a)

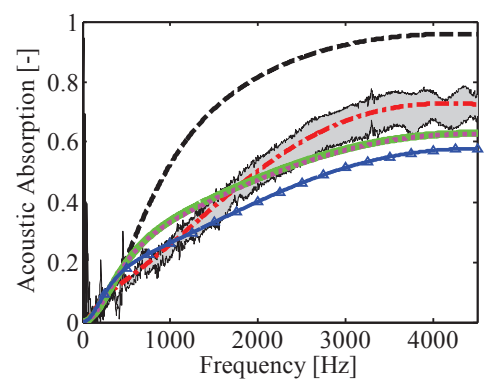

(b)

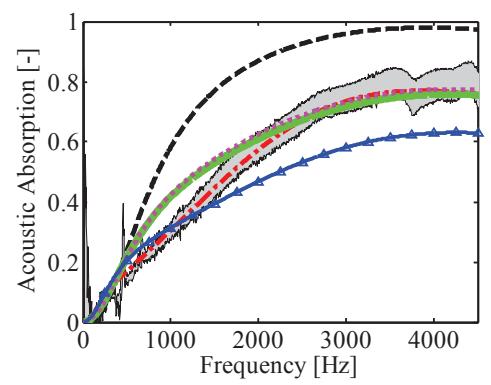

(c)

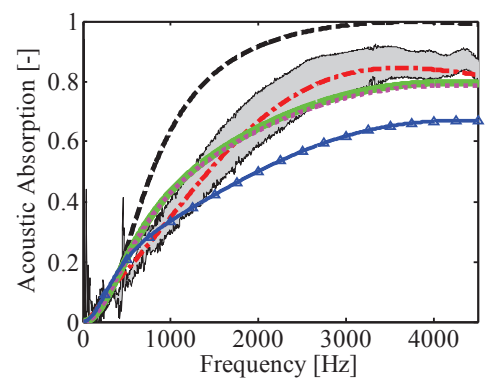

(d)

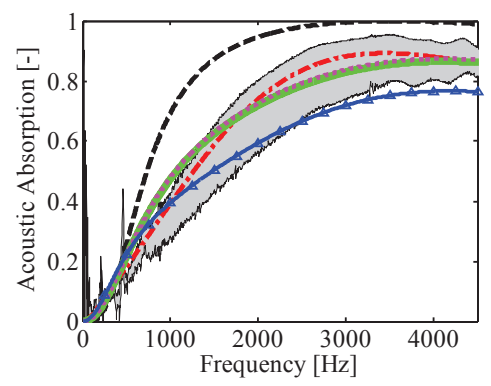

(e)

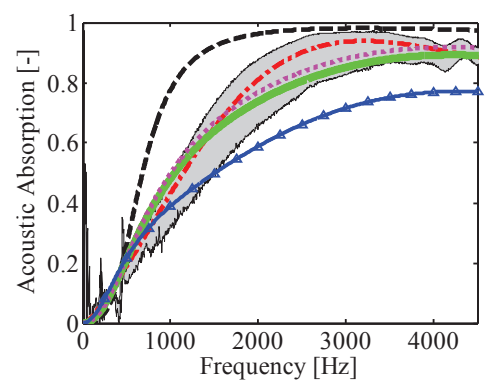

(f)

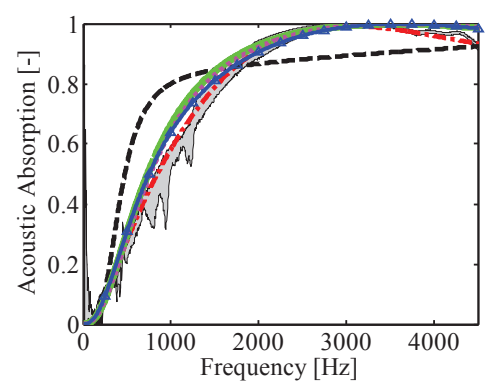

(g)

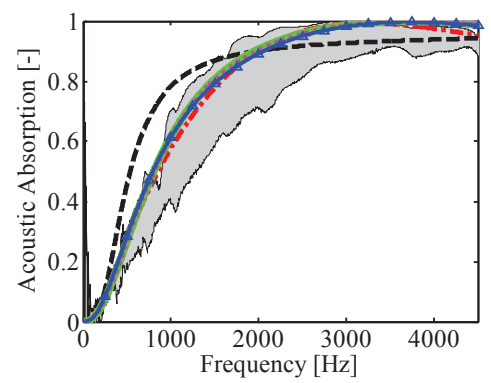

(h)

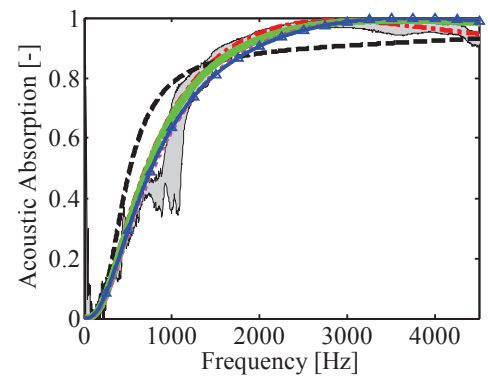

(i)

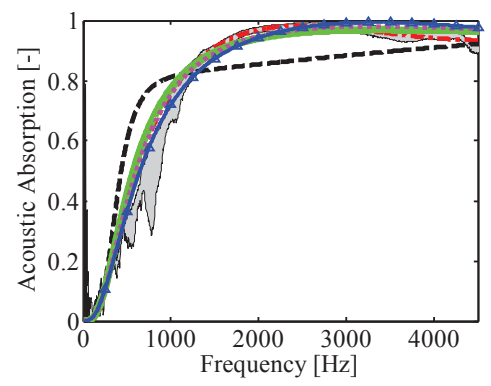

(j)

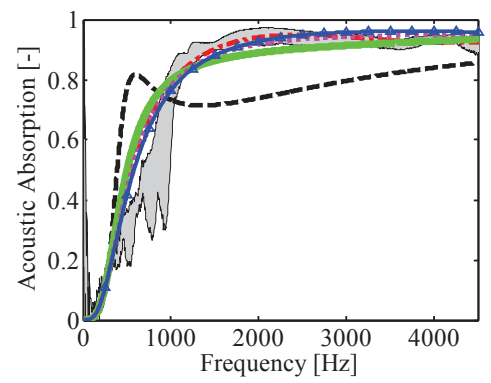

FIG. 18. (Color online) Sound absorption coefficient (normal incidence). (a)-(e): Product 1 to 5 (from top to bottom). (f)-(j): Product 6 to 10 (from top to bottom). Colors and symbols: measurements (gray zone); direct and inverse characterizations (dash-dot line, red); equivalent microstructure $(\triangle$, solid line, blue); random microstructure with diameters distributed according to a Gamma law - PDFM (dashed line, black); random microstructure with weighted diameters PDFM-WD (thick line, green); random microstructure with constant fiber diameter - MDFM-WD (dotted line, magenta). 


\section{CONCLUSION}

A recent approach has been to treat a polydisperse fibrous media as if it was equivalent to a monodisperse fibrous media, i.e. by means of an average diameter of the corresponding distribution [24]. Here, the effective fiber diameter is derived from optical granulomorphometry and is a weighted fiber volume diameter accounting for the relative length and diameter of each fiber. We have first addressed the definition of an equivalent microstructure comprising of a three-dimensional network of cylindrical elements, characterized by a weighted fiber volume diameter and a preferred out-of-plane orientation. After rescaling the domain characteristic lengths and surface areas to those of the real glass wool sample under study with a preserved experimental porosity, the thermal and viscous characteristic lengths computed from the reconstructed fiber webs are then multiplied by a compensation factor. This factor accounts for the experimental distributions of both the fiber diameter and length, and it should be noticed that there is no free parameter in such a procedure. Stochastic models for random microstructures were subsequently introduced in a classical manner. It was shown that the models PDFM-WD and MDFM-WD provide an excellent estimate of the through-thickness viscous permeability. The predictions of the viscous characteristic length were found less reliable as compared with experiments, especially at low density (very high porosity). The sound absorption was accurately simulated for all the glass wool products under study, which correspond to various processing parameters. This range of applicability makes the proposed multiscale framework attractive for the prediction of sound absorbing properties for complex fibrous materials.

\section{ACKNOWLEDGMENTS}

We thank Y. Heulin and M. Daudet from Isover Saint-Gobain CRIR who performed the acoustical and morphogranulometry characterizations, respectively; and for discussions about the data analysis. P. André, S. Berger, J.-B. Chéné, C. Coguenanff, E. Thibier from the thesis committee are gratefully acknowledged for providing insightful discussions and suggestions. This work was supported by the French Agency for the Environment and Energy Management (ADEME), the French Scientific and Technical Center for Building (CSTB), and the Isover Saint-Gobain Company, under the cooperative agreement ADEME 
TEZ14-24 with the University Paris-Est Marne-la-Vallée.

[1] E. J. Garboczi, "Permeability, diffusivity, and microstructural parameters: A critical review," Cem. Concr. Res. 20, 591-601 (1990).

[2] P. M. Adler and J.-F. Thovert, "Real porous media: Local geometry and macroscopic properties," Appl. Mech. Rev. 51, 537-585 (1998).

[3] M. E. Delany and E. N. Bazley, "Acoustical properties of fibrous materials," Appl. Acoust. 3, 105-116 (1970).

[4] D. A. Bies and C. H. Hansen, "Flow resistance information for acoustical design," Appl. Acoust. 13, 357-391 (1980).

[5] Y. Miki, "Acoustical properties of porous materials - modifications of Delany Bazley models," J. Acoust. Soc. Jpn. (E) 11, 19-24 (1990).

[6] M. Garai and F. Pompoli, "A simple empirical model of polyester fibre materials for acoustical applications," Appl. Acoust. 66, 1383-1398 (2005).

[7] J. Manning and R. Panneton, "Acoustical model for Shoddy-based fiber sound absorbers," Text. Res. J. 83, 1356-1370 (2013).

[8] P. Kerdudou, J.-B. Chéné, G. Jacqus, C. Perrot, S. Berger, P. Leroy, "A semi-empirical approach to link macroscopic parameters to microstructure of fibrous materials," in Proceedings of the 44th InterNoise (San Francisco, CA, 2015), pp. 5468-5479.

[9] J. L. Auriault, L. Borne, and R. Chambon, "Dynamics of porous saturated media, checking of the generalized law of Darcy," J. Acoust. Soc. Am. 7r, 1641-1650 (1985).

[10] D. L. Johnson, J. Koplik, R. Dashen, "Theory of dynamic permeability and tortuosity in fluid-saturated porous media," J. Fluid Mech. 176, 379-402 (1987).

[11] Y. Champoux, J. F. Allard, "Dynamic tortuosity and bulk modulus in air-saturated porous media," J. Appl. Phys. 70, 1975-1979 (1991).

[12] S. R. Pride, F. D. Morgan, A. F. Gangi, "Drag forces of porous media acoustics," Phys. Rev. B 47, 4964-4978 (1993).

[13] D. Lafarge, P. Lemarinier, J.-F. Allard, V. Tarnow, "Dynamic compressibility of air in porous structures at audible frequencies," J. Acoust. Soc. Am. 102, 1995-2006 (1997). 
[14] C. Boutin, "Rayleigh scattering of acoustic waves in rigid porous media," J. Acoust. Soc. Am. 122, 1888-1905 (2007).

[15] K. Schladitz, S. Peters, D. Reinel-Bitzer, A. Wiegmann, J. Ohser, "Design of acoustic trim based on geometric modeling and flow simulation for non-woven," Comp. Mat. Sci. 38, 56-66 (2006).

[16] C. Peyrega and D. Jeulin, "Estimation of acoustic properties and of the representative volume element of random fibrous media," J. Appl. Phys. 113, 104901-13 (2013).

[17] H. T. Luu, C. Perrot, V. Monchiet, R. Panneton, "Three-dimensional reconstruction of a random fibrous medium: Geometry, transport and sound absorbing properties," J. Acoust. Soc. Am. 141, 4768-4780 (2017).

[18] O. Umnova, D. Tsiklauri, R. Venegas, "Effect of boundary slip on the acoustical properties of microfibrous materials," J. Acoust. Soc. Am. 126, 1850-1861 (2009).

[19] V. Tarnow, "Compressibility of air in fibrous materials," J. Acoust. Soc. Am. 99, 3010-3017 (1996).

[20] V. Tarnow, "Airflow resistivity of models of fibrous acoustic materials," J. Acoust. Soc. Am. 100, 3706-3713 (1996).

[21] B. P. Semeniuk and P. Göransson, "Microstructure based estimation of the dynamic drag impedance of lightweight fibrous materials," J. Acoust. Soc. Am. 141, 1360-1370 (2017).

[22] M. M. Tomadakis and T. J. Robertson, "Viscous permeability of random fiber structures: Comparison of electrical and diffusional estimates with experimental and analytical results," J. Comp. Mat. 39, 163-188 (2005).

[23] H. T. Luu, R. Panneton, C. Perrot, "Effective fiber diameter for modeling the acoustic properties of polydisperse fiber networks," J. Acoust. Soc. Am. 141, EL96-EL101 (2017).

[24] H. T. Luu, C. Perrot, R. Panneton, "Infuence of porosity, fiber radius and fiber orientation on the transport and acoustic properties of random fiber structures," Acta Acust. united Ac., 103, 1050-1063 (2017).

[25] C. Jensen and R. Raspet, "Thermoacoustic properties of fibrous materials," J. Acoust. Soc. Am. 127, 3470-3484 (2010).

[26] P. Lallemand and L. S. Luo, "Theory of the lattice Boltzmann method: Acoustics and thermal properties in two and three dimensions," Phys. Rev. E 68, 036706-25 (2003). 
[27] K. Attenborough, "Acoustical characteristics of rigid fibrous absorbents and granular materials," J. Acoust. Soc. Am. 73, 785-799 (1983).

[28] C. Zwikker and C. W. Kosten, Sound Absorbing Materials, (Elsevier, New York, 1949), pp. 174.

[29] D. K. Wilson, "Relaxation-matched modeling of propagation through porous media, including fractal pore structure," J. Acoust. Soc. Am. 94, 1136-1145 (1993).

[30] C. Peyrega, D. Jeulin, C. Delise, J. Malvestio, "3D morphological characterization of phonic insulation fibrous media," Adv. Eng. Mater. 13, 156-164 (2010).

[31] H. Talbot, "Analyse morphologique de fibres minerals d'isolation," Ph. D. thesis (translation in English: "Morphological analysis of mineral insulation fibers"), Ecole Nationale Supérieure des Mines de Paris, 1993.

[32] S. Bergonnier, "Relationship between microstructure and mechanical properties of entangled materials," Ph. D. thesis, Université Pierre et Marie Curie - Paris VI, 2005; translation in English: "Morphological analysis of mineral insulation fibers".

[33] L. Chapelle, "Characterization and modelling of the mechanical properties of mineral wool," Ph. D. thesis, Technical University of Denmark, 2016.

[34] M. D. Abramoff, P. J. Magalhaes, S. J. Ram, "Image Processing with ImageJ," Biophotonics Int. 11, 36-42 (2004).

[35] Y. Salissou, R. Panneton, "Pressure/mass method to measure open porosity of porous solids," J. Appl. Phys. 101, 124913-7 (2007).

[36] M. R. Stinson and G. A. Daigle, "Electronic system for the measurement of flow resistance," J. Acoust. Soc. Am. 83, 2422-2428 (1988).

[37] Acoustics - Materials for acoustical applications - Determination of airflow resistance, ISO 9053 (1991).

[38] R. Panneton and X. Olny, "Acoustical determination of the parameters governing viscous dissipation in porous media," J. Acoust. Soc. Am. 119, 2027-2040 (2006).

[39] X. Olny and R. Panneton, "Acoustical determination of the parameters governing thermal dissipation in porous media," J. Acoust. Soc. Am. 123, 814-824 (2008).

[40] Y. Salissou and R. Panneton, "Wideband characterization of complex wave number and characteristic impedance of sound absorbers," J. Acoust. Soc. Am. 128, 2868-2876 (2010). 
[41] O. Doutres, Y. Salissou, N. Atalla, R. Panneton, "Evaluation of the acoustic and nonacoustic properties of sound absorbing materials using a three-microphone impedance tube," Appl. Acoust. 71, 506-509 (2010).

[42] R. Panneton, "Comments on the limp frame equivalent fluid model for porous media," J. Acoust. Soc. Am. 122, EL217-EL222 (2007).

[43] L. Dormieux, D. Kondo, F.-J. Ulm, Microporomechanics (Wiley, Chichester, 2006), pp. 344.

[44] A. Papoulis and S.U. Pillai, Probability, random variables, and stochastic processes (McGrawHill, New-York, 2002), pp. 82.

[45] J.-L. Auriault, C. Boutin, C. Geindreau, Homogenization of Coupled Phenomena in Heterogenous Media (Wiley-ISTE, London, 2009), pp. 476.

[46] A. Ern and J.-L. Guermond, Theory and Practice of Finite Elements (Springer, New-York, 2004), pp. 505.

[47] D. N. Arnold, F. Brezzi, M. Fortin, "A stable finite element for Stokes equations," Calcolo 21, 337-344 (1984).

[48] C. Sandström and F. Larsson, "On bounded approximations of periodicity for computational homogenization of Stokes flow in porous media," Int. J. Numer. Methods Eng. 109, 307-325 (2016).

[49] A. E. Scheidegger, The physics of flow through porous media (University of Toronto Press, Toronto, 1957), pp. 7.

[50] L. M. Schwartz, N. Martys, D. P. Bentz, E. J. Garboczi, and S. Torquato, "Cross-property relations and permeability estimation in model porous media," Phys. Rev. E 48, 4584-4591 (1993).

[51] F. M. Auzerais, J. Dunsmuir, B. B. Ferréol, N. Martys, J. Olson, T. S. Ramakrishnan, D. H. Rothman, and L. M. Schwartz, "Transport in sandstone: A study based on three dimensional microtomography," Geophys. Res. Lett. 23, 705-708 (1996).

[52] J. Guilleminot, C. Soize, R. G. Ghanem, "Stochastic representation for anisotropic permeability tensor random fields," Int. J. Numer. Anal. Methods Geomech. 36, 1592-1608 (2012). 